TRANSACTIONS OF THE

AMERICAN MATHEMATICAL SOCIETY

Volo 351 Nun 6 , Pages $2407-2438$

S 0002-9947(99)02449-6

Article electronically published on February 15, 1999

\title{
MULTIVARIATE MATRIX REFINABLE FUNCTIONS WITH ARBITRARY MATRIX DILATION
}

\author{
QINGTANG JIANG
}

\begin{abstract}
Characterizations of the stability and orthonormality of a multivariate matrix refinable function $\Phi$ with arbitrary matrix dilation $M$ are provided in terms of the eigenvalue and 1-eigenvector properties of the restricted transition operator. Under mild conditions, it is shown that the approximation order of $\Phi$ is equivalent to the order of the vanishing moment conditions of the matrix refinement mask $\left\{\mathbf{P}_{\alpha}\right\}$. The restricted transition operator associated with the matrix refinement mask $\left\{\mathbf{P}_{\alpha}\right\}$ is represented by a finite matrix $\left(\mathcal{A}_{M i-j}\right)_{i, j}$, with $\mathcal{A}_{j}=|\operatorname{det}(M)|^{-1} \sum_{\kappa} \mathbf{P}_{\kappa-j} \otimes \mathbf{P}_{\kappa}$ and $\mathbf{P}_{\kappa-j} \otimes \mathbf{P}_{\kappa}$ being the Kronecker product of matrices $\mathbf{P}_{\kappa-j}$ and $\mathbf{P}_{\kappa}$. The spectral properties of the transition operator are studied. The Sobolev regularity estimate of a matrix refinable function $\Phi$ is given in terms of the spectral radius of the restricted transition operator to an invariant subspace. This estimate is analyzed in an example.
\end{abstract}

\section{INTRODUCTION}

Let $\left\{\mathbf{P}_{\alpha}\right\}$ be a finitely supported $r \times r$ matrix sequence. The vectors $\Phi, r$ dimensional column functions on $\mathbb{R}^{d}$, considered in this paper are solutions to functional equations of the type

$$
\Phi=\sum_{\alpha \in \mathbb{Z}^{d}} \mathbf{P}_{\alpha} \Phi(M \cdot-\alpha),
$$

where $M$ is a $d \times d$ integer matrix with $m=|\operatorname{det}(M)| \geq 2$ and all eigenvalues of modulus $>1$. Define

$$
\mathbf{P}(\omega):=\frac{1}{m} \sum_{\alpha \in \mathbb{Z}^{d}} \mathbf{P}_{\alpha} \exp (-i \alpha \omega) .
$$

Then $\mathbf{P}$ is an $r \times r$ matrix with trigonometric polynomial entries. In the Fourier domain, functional equations (1.1) can be written as

$$
\widehat{\Phi}(\omega)=\mathbf{P}\left({ }^{t} M^{-1} \omega\right) \widehat{\Phi}\left({ }^{t} M^{-1} \omega\right) .
$$

Throughout this paper, ${ }^{t} A$ and $A^{*}$ denote the transpose and the Hermitian adjoint of a matrix $A$ respectively.

Received by the editors September 26, 1996.

1991 Mathematics Subject Classification. Primary 39B62, 42B05, 41A15; Secondary 42C15.

Key words and phrases. Matrix refinable function, transition operator, stability, orthonormality, approximation order, regularity.

The author was supported by an NSTB post-doctoral research fellowship at the National University of Singapore. 
Equations of type (1.1) or (1.2) are called matrix (vector) refinement equations; the matrix $M$ is called the dilation matrix; $\mathbf{P}\left(\left\{\mathbf{P}_{\alpha}\right\}\right)$ is called the (matrix) refinement mask and any solution $\Phi$ of (1.1) is called an $(M, \mathbf{P})$ matrix refinable function (or an $(M, \mathbf{P})$ refinable vector).

For $M=2 \mathbf{I}_{r}, r \geq 1$, where $\mathbf{I}_{r}$ is the $r \times r$ identity matrix, the characterizations of the stability and orthonormality of a matrix refinable function $\Phi$ were provided in terms of the mask in [26]; the regularity estimates of $\Phi$ were studied in [26], [19], and in [3], [24] for the case $d=1$; the existence of the distribution solution of (1.1) and the characterization of the weak stability of solutions of (1.1) were discussed in [21]. In the construction of multivariate wavelets, the dilation matrix $M$ is involved. For $r=1$, the characterizations of the stability and orthonormality of $\Phi$, a refinable function with matrix dilation, were proved in terms of the mask in [22]; the optimal Sobolev regularity estimate of $\Phi$ was obtained in [15]. Our goal in this paper is to provide characterizations of the stability, orthonormality and the approximation order of an $(M, \mathbf{P})$ refinable vector $\Phi$ in terms of the mask, and give the regularity estimate of $\Phi$ in terms of the spectral radius of the restricted transition operator.

Before going further, we introduce some notations used in this paper. Let $\mathbb{Z}_{+}$ denote the set of all nonnegative integers, and let $\mathbb{Z}_{+}^{d}$ denote the set of all $d$-tuples of nonnegative integers. We shall adopt the multi-index notations

$$
\omega^{\beta}:=\omega_{1}^{\beta_{1}} \cdots \omega_{d}^{\beta_{d}}, \quad \beta !:=\beta_{1} ! \cdots \beta_{d} !, \quad|\beta|:=\beta_{1}+\cdots+\beta_{d}
$$

for $\omega={ }^{t}\left(\omega_{1}, \cdots, \omega_{d}\right) \in \mathbb{R}^{d}, \beta={ }^{t}\left(\beta_{1}, \cdots, \beta_{d}\right) \in \mathbb{Z}_{+}^{d}$. If $\alpha, \beta \in \mathbb{Z}^{d}$ satisfy $\beta-\alpha \in \mathbb{Z}_{+}^{d}$, we shall write $\alpha \leq \beta$ and denote

$$
\left(\begin{array}{c}
\beta \\
\alpha
\end{array}\right):=\frac{\beta !}{\alpha !(\beta-\alpha) !} .
$$

For $\beta={ }^{t}\left(\beta_{1}, \cdots, \beta_{d}\right) \in \mathbb{Z}_{+}^{d}$, denote

$$
D^{\beta}:=\frac{\partial^{\beta_{1}}}{\partial x_{1}^{\beta_{1}}} \cdots \frac{\partial^{\beta_{d}}}{\partial x_{d}^{\beta_{d}}},
$$

where $\partial_{j}=\frac{\partial}{\partial x_{j}}$ is the partial derivative operator with respect to the $j$ th coordinate, $1 \leq j \leq d$. Except in some special cases, for $\omega, \zeta \in \mathbb{R}^{d}$ we use $\zeta \omega$ (not ${ }^{t} \zeta \omega$ ) to denote their scalar product.

For a finitely supported complex sequence $c$ on $\mathbb{Z}^{d}$, its support is defined by $\operatorname{supp} c:=\left\{\beta \in \mathbb{Z}^{d}: c(\beta) \neq 0\right\}$, and for a finitely supported $r \times r$ matrix sequence $C$ on $\mathbb{Z}^{d}$, its support is defined by $\operatorname{supp} C:=\bigcup \operatorname{supp} c_{i j}$, where $c_{i j}$ is the $(i, j)$-entry of $C$. Throughout this paper, we assume that the matrix refinement mask $\mathbf{P}$ satisfies $\operatorname{supp}\left\{\mathbf{P}_{\alpha}\right\} \subset[0, N]^{d}$ for some positive integer $N$.

Let $\|x\|$ denote the Euclidean norm in $\mathbb{R}^{d}$, and let $\operatorname{dist}(x, y):=\|x-y\|$ be the distance between two points $x, y \in \mathbb{R}^{d}$. For two subsets $S_{1}, S_{2}$ of $\mathbb{R}^{d}$, denote

$$
\operatorname{dist}\left(S_{1}, S_{2}\right):=\inf \left\{\operatorname{dist}(x, y): x \in S_{1}, y \in S_{2}\right\} .
$$

For any subset $S$ of $\mathbb{R}^{d}$, denote $[S]:=S \cap \mathbb{Z}^{d}$; and if $S$ is a finite set of $\mathbb{Z}^{d}$, let $|S|$ denote the number of elements in $S$.

For $j=1, \cdots, r$, let $\mathbf{e}_{j}:=\left(\delta_{j}(k)\right)_{k=1}^{r}$ denote the standard unit vectors in $\mathbb{R}^{r}$. In this paper, for an $r \times 1$ vector-valued function or sequence $f={ }^{t}\left(f_{1}, \cdots, f_{r}\right)$, when we say that $f$ is in a space on $\mathbb{R}^{d}$ or $\mathbb{Z}^{d}$, we mean that every component $f_{i}$ of $f$ is in 
this space. In particular, $f={ }^{t}\left(f_{1}, \cdots, f_{r}\right) \in L^{2}\left(\mathbb{R}^{d}\right)\left(\right.$ or $\left.\mathbf{c}=\left(c_{1}, \cdots, c_{r}\right) \in l^{2}\left(\mathbb{Z}^{d}\right)\right)$ means that $f_{i} \in L^{2}\left(\mathbb{R}^{d}\right)$ (or $\left.c_{i} \in l^{2}\left(\mathbb{Z}^{d}\right)\right), i=1, \cdots, r$, and we will use the norms

$$
\|f\|_{2}=\left(\sum_{i=1}^{r}\left\|f_{i}\right\|_{L^{2}\left(\mathbb{R}^{d}\right)}^{2}\right)^{\frac{1}{2}}, \quad\|\mathbf{c}\|_{2}=\left(\sum_{i=1}^{r}\left\|c_{i}\right\|_{l^{2}\left(\mathbb{Z}^{d}\right)}^{2}\right)^{\frac{1}{2}} .
$$

For a matrix $A$ (or an operator $A$ defined on a finite dimensional linear space), we say $A$ satisfies Condition $\mathbf{E}$ if $\rho(A) \leq 1,1$ is the unique eigenvalue on the unit circle and 1 is simple (the spectral radius of $A$ is denoted by $\rho(A)$ ).

Let $M$ be a fixed dilation matrix with $m=|\operatorname{det}(M)|$. Then the coset spaces $\mathbb{Z}^{d} /\left(M \mathbb{Z}^{d}\right)$ and $\mathbb{Z}^{d} /\left({ }^{t} M \mathbb{Z}^{d}\right)$ consist of $m$ elements. Let $\gamma_{k}+M \mathbb{Z}^{d}, 1 \leq k \leq m-1$, and $\eta_{j}+{ }^{t} M \mathbb{Z}^{d}, j=0, \cdots, m-1$, be the $m$ distinct elements of $\mathbb{Z}^{d} /\left(M \mathbb{Z}^{d}\right)$ and $\mathbb{Z}^{d} /\left({ }^{t} M \mathbb{Z}^{d}\right)$ respectively, with $\gamma_{0}=0, \eta_{0}=0$. Let $C_{0}\left(\mathbb{T}^{d}\right)$ denote the space of all $r \times r$ matrix functions with trigonometric polynomial entries. For a given matrix refinement mask $\mathbf{P}$, the transition operator $\mathbf{T}$ associated with $\mathbf{P}$ is defined on $C_{0}\left(\mathbb{T}^{d}\right)$ by

$$
\mathbf{T} C(\omega):=\sum_{j=0}^{m-1} \mathbf{P}\left({ }^{t} M^{-1}\left(\omega+2 \pi \eta_{j}\right)\right) C\left({ }^{t} M^{-1}\left(\omega+2 \pi \eta_{j}\right)\right) \mathbf{P}^{*}\left({ }^{t} M^{-1}\left(\omega+2 \pi \eta_{j}\right)\right) .
$$

Assume that the support of the mask $\left\{\mathbf{P}_{\alpha}\right\}$ is in $[0, N]^{d}$, and denote

$$
\Omega:=\left\{\sum_{j=0}^{\infty} M^{-(j+1)} x_{j}: x_{j} \in[-N, N]^{d}, \forall j \in \mathbb{Z}_{+}\right\} .
$$

Let $\mathbb{H}$ denote the subspace of $C_{0}\left(\mathbb{T}^{d}\right)$ defined by

$$
\mathbb{H}:=\left\{H(\omega) \in C_{0}\left(\mathbb{T}^{d}\right): H(\omega)=\sum_{\alpha} H_{\alpha} e^{-i \alpha \omega}, \operatorname{supp}\left\{H_{\alpha}\right\} \subset[\Omega]\right\} .
$$

Recall that a vector-valued function $\Psi={ }^{t}\left(\psi_{1}, \cdots, \psi_{r}\right)$ is called stable (orthogonal) if the integer translates of $\psi_{1}, \cdots, \psi_{r}$ form a Riesz basis (an orthonormal basis) of their closed linear span in $L^{2}(\mathbb{R})$. It has been shown that an $(M, \mathbf{P})$ refinable vector $\Phi$ is stable if and only if for all $\omega \in \mathbb{T}^{d}, G_{\Phi}(\omega) \geq c \mathbf{I}_{r}$ for some positive constant $c$, and that $\Phi$ is orthogonal if and only if $G_{\Phi}(\omega)=\mathbf{I}_{r}, \omega \in \mathbb{T}^{d}$; see e.g. [6], [10], [16] and [23]. Here $G_{\Phi}(\omega)$ is the Gram matrix of $\Phi$, defined by

$$
G_{\Phi}(\omega):=\sum_{\alpha \in \mathbb{Z}^{d}} \widehat{\Phi}(\omega+2 \pi \alpha) \widehat{\Phi}^{*}(\omega+2 \pi \alpha) .
$$

In the first part of Section 2, we will show that if the refinement equation (1.1) has a compactly supported solution $\Phi$ such that $G_{\Phi}(\omega)<\infty$ and $\operatorname{det}\left(G_{\Phi}(0)\right) \neq 0$, then $\mathbf{P}(0)$ satisfies Condition E. Then we will provide a characterization of the existence of $L^{2}$-solutions of (1.1) under the assumption that $\mathbf{P}(0)$ satisfies Condition E. In the last part of Section 2, we will show that the $(M, \mathbf{P})$ refinable vector $\Phi$ is stable if and only if the restriction $\left.\mathbf{T}\right|_{\mathbb{H}}$ of the transition operator $\mathbf{T}$ to $\mathbb{H}$ satisfies Condition $\mathrm{E}$ and the corresponding 1-eigenvector of $\left.\mathbf{T}\right|_{\mathbb{H}}$ is positive (or negative) definite on $\mathbb{T}^{d}$, and show that the $(M, \mathbf{P})$ refinable vector $\Phi$ is orthogonal if and only if $\left.\mathbf{T}\right|_{\mathbb{H}}$ satisfies Condition E and $\mathbf{P}$ is a Conjugate Quadrature Filter (CQF), i.e.

$$
\sum_{j=0}^{m-1} \mathbf{P}\left({ }^{t} M^{-1}\left(\omega+2 \pi \eta_{j}\right)\right) \mathbf{P}^{*}\left({ }^{t} M^{-1}\left(\omega+2 \pi \eta_{j}\right)\right)=\mathbf{I}_{r}, \quad \omega \in \mathbb{T}^{d} .
$$


The accuracy order of the $(M, \mathbf{P})$ refinable vector $\Phi={ }^{t}\left(\phi_{1}, \cdots, \phi_{r}\right)$ was considered in [11], [25] and [17] for the case $d=1$ and $M=(2)$, in [7] for $M=2 \mathbf{I}_{r}$ and in [1] for the multivariate case with arbitrary dilation matrix. In Section 3, we will show that, under mild conditions, $\Phi$ provides approximation of order $k$, $k \in \mathbb{Z}_{+} \backslash\{0\}$, if and only if the matrix refinement mask $\mathbf{P}$ satisfies the vanishing moment conditions of order $k$. We will also determine explicitly the coefficients for the polynomial reproducing under the assumption that the integer shifts of $\Phi$ $\left(\phi_{l}(\cdot-\kappa), \kappa \in \mathbb{Z}^{d}, l=1, \cdots, r\right)$ are linearly independent.

Since the spectra (eigenvalues) of a matrix can be computed directly, it is useful in practice to transfer equivalently the restricted operator $\left.\mathbf{T}\right|_{\mathbb{H}}$ to be a finite matrix, and therefore transfer the spectral problems of $\left.\mathbf{T}\right|_{\mathbb{H}}$ into those of a matrix. We will show in Section 4 that the restricted transition operator $\left.\mathbf{T}\right|_{\mathbb{H}}$ is equivalent to the matrix $\left(\mathcal{A}_{M i-j}\right)_{i, j \in[\Omega]}$, where $\mathcal{A}_{j}$ is the $r^{2} \times r^{2}$ matrix given by

$$
\mathcal{A}_{j}=\frac{1}{|\operatorname{det}(M)|} \sum_{\kappa \in[0, N]^{d}} \mathbf{P}_{\kappa-j} \otimes \mathbf{P}_{\kappa}
$$

and $\mathbf{P}_{\kappa-j} \otimes \mathbf{P}_{\kappa}$ is the Kronecker product of $\mathbf{P}_{\kappa-j}$ and $\mathbf{P}_{\kappa}$. We will also consider the spectral property of $\mathbf{T}$ in Section 4.

In the last part of this paper, Section 5, we will consider the regularity of the $(M, \mathbf{P})$ refinable vector $\Phi$. An invariant subspace $\mathbb{H}^{0}$ of $\mathbb{H}$ under $\mathbf{T}$ is found, and it is shown that $\Phi$ is in the Sobolev space $W^{s_{0}-\epsilon}\left(\mathbb{R}^{d}\right)$ for any $\epsilon>0$, where $s_{0}:=$ $-\log \rho\left(\left.\mathbf{T}\right|_{\mathbb{H}^{0}}\right) /\left(2 \log \lambda_{\max }\right), \rho\left(\left.\mathbf{T}\right|_{\mathbb{H}^{0}}\right)$ is the spectral radius of the restriction $\mathbf{T}_{\mathbb{H}^{0}}$ of $\mathbf{T}$ to $\mathbb{H}^{0}$ and $\lambda_{\max }$ is the spectral radius of the dilation matrix $M$. This estimate is analyzed in an example.

\section{Stability AND ORTHONORMALity}

In this section, we will provide characterizations of the stability and orthonormality of the refinable vector $\Phi$. We first prove some lemmas.

Lemma 2.1. Let $\gamma_{k}+M \mathbb{Z}^{d}, 1 \leq k \leq m-1$, and $\eta_{j}+{ }^{t} M \mathbb{Z}^{d}, j=0, \cdots, m-1$, be the $m$ distinct elements of the coset spaces $\mathbb{Z}^{d} /\left(M \mathbb{Z}^{d}\right)$ and $\mathbb{Z}^{d} /\left({ }^{t} M \mathbb{Z}^{d}\right)$ respectively, with $\gamma_{0}=0, \eta_{0}=0$. Then

$$
\begin{array}{ll}
\sum_{k=0}^{m-1} e^{i 2 \pi^{t} \eta_{j} M^{-1} \gamma_{k}}=m \delta(j), & 0 \leq j \leq m-1 \\
\sum_{j=0}^{m-1} e^{i 2 \pi^{t} \eta_{j} M^{-1} \gamma_{k}}=m \delta(k), & 0 \leq k \leq m-1 .
\end{array}
$$

Proof. Let $G$ be the finite abelian group consisting of $\gamma_{k}+M \mathbb{Z}^{d}, 1 \leq k \leq m-1$. For any $j, 0 \leq j \leq m-1$, define on $G$ the functions $\chi_{j}(g):=e^{i 2 \pi^{t} \eta_{j} M^{-1} g}, g \in G$. Then $\chi_{j}(g), j=0, \cdots, m-1$, form the group $\widehat{G}$, the character group of $G$. By the orthonormality relation of characters (see [4]), we have

$$
\sum_{k=0}^{m-1} \chi_{j}(g) \overline{\chi_{j^{\prime}}(g)}=m \delta_{j}\left(j^{\prime}\right), \quad 0 \leq j, j^{\prime} \leq m-1 .
$$

Let $j^{\prime}=0$; then (2.3) leads to (2.1). Since the transpose of ${ }^{t} M$ is $M,(2.2)$ follows from $(2.1)$. 
Let $\Omega$ denote the domain defined by (1.4) and denote

$$
\Omega_{+}:=\left\{\sum_{j=0}^{\infty} M^{-(j+1)} x_{j}: \quad x_{j} \in[0, N]^{d}, \forall j \in \mathbb{Z}_{+}\right\} .
$$

The proof of the following lemma can be carried out by modifying that of Lemma 3.1 in [15] for the case $r=1$.

Lemma 2.2. Assume that $\operatorname{supp}\left\{\mathbf{P}_{\alpha}\right\} \subset[0, N]^{d}$ and $\Phi$ is a compactly supported $(M, \mathbf{P})$ matrix refinable function. Let $\mathbf{T}$ be the transition operator defined by (1.3) and $\mathbb{H}$ the space defined by (1.5). Then

(i) $\operatorname{supp} \Phi \subset \Omega_{+}$,

(ii) $\mathbb{H}$ is invariant under $\mathbf{T}$,

(iii) for any $C(\omega) \in C_{0}\left(\mathbb{T}^{d}\right)$, there exists some $n \in \mathbb{Z}_{+}$such that $\mathbf{T}^{n} C \in \mathbb{H}$,

(iv) the eigenvectors of $\mathbf{T}$ corresponding to nonzero eigenvalues belong to $\mathbb{H}$.

Proof. (i) can be obtained similarly to Lemma 3.1 in [15]. Here we verify (ii), (iii) and (iv).

For any $H=\sum_{\ell \in \mathbb{Z}^{d}} H_{\ell} e^{-i \ell \omega} \in C_{0}\left(\mathbb{T}^{d}\right)$, one has

$$
\mathbf{P}(\omega) H(\omega) \mathbf{P}^{*}(\omega)=m^{-2} \sum_{\ell \in \mathbb{Z}^{d}} \sum_{\kappa \in[0, N]^{d}} \sum_{n \in \mathbb{Z}^{d}} \mathbf{P}_{\kappa} H_{\ell}{ }^{t} \mathbf{P}_{\kappa-n} e^{-i \omega(n+\ell)} .
$$

Thus

$$
\mathbf{T} H(\omega)=m^{-2} \sum_{j=0}^{m-1} \sum_{\ell \in \mathbb{Z}^{d}} \sum_{n \in \mathbb{Z}^{d}} \sum_{\kappa \in[0, N]^{d}} \mathbf{P}_{\kappa} H_{\ell}{ }_{\ell} \mathbf{P}_{\kappa-n} e^{-i\left({ }^{t} M^{-1}\left(\omega+2 \pi \eta_{j}\right)\right)(n+\ell)} .
$$

For any $n \in \mathbb{Z}^{d}, \ell \in \mathbb{Z}^{d}$, write $n+\ell=M \tau+\gamma_{k}$ for some $\tau \in \mathbb{Z}^{d}$ and $k \in \mathbb{Z}_{+}, 0 \leq$ $k \leq m-1$. By Lemma 2.1,

$$
\mathbf{T} H(\omega)=m^{-1} \sum_{\tau \in \mathbb{Z}^{d}}\left(\sum_{\ell \in \mathbb{Z}^{d}} \sum_{\kappa \in[0, N]^{d}} \mathbf{P}_{\kappa} H_{\ell}{ }^{t} \mathbf{P}_{\kappa-(M \tau-\ell)}\right) e^{-i \omega \tau} .
$$

If $H \in \mathbb{H}$, then $H=\sum_{\ell \in[\Omega]} H_{\ell} e^{-i \ell \omega}$ and

$$
\mathbf{T} H(\omega)=m^{-1} \sum_{\tau \in \mathbb{Z}^{d}} \sum_{\ell \in[\Omega]} \sum_{\kappa \in[0, N]^{d}} \mathbf{P}_{\kappa} H_{\ell}{ }^{t} \mathbf{P}_{\kappa-(M \tau-\ell)} e^{-i \omega \tau} .
$$

If $\mathbf{T} H(\omega) \neq 0$, then $M \tau-\ell \in[-N, N]^{d}$ for some $\ell \in[\Omega]$, i.e. $\mathbf{M} \tau \in[-N, N]^{d}+\Omega$. Thus $\tau \in M^{-1}[-N, N]^{d}+M^{-1} \Omega=\Omega$, and we have

$$
\mathbf{T} H(\omega)=m^{-1} \sum_{\tau \in[\Omega]}\left(\sum_{\ell \in[\Omega]} \sum_{\kappa \in[0, N]^{d}} \mathbf{P}_{\kappa} H_{\ell}{ }^{t} \mathbf{P}_{\kappa-(M \tau-\ell)}\right) e^{-i \omega \tau} .
$$

Hence $\mathbb{H}$ is invariant under $\mathbf{T}$.

For $C \in C_{0}\left(\mathbb{T}^{d}\right)$ and $j \in \mathbb{Z}_{+}$, denote $\mathbf{T}^{j} C=: \sum_{\tau \in \mathbb{Z}^{d}} C^{(j)}(\tau) e^{-i \omega \tau}$. By (2.4),

$$
\operatorname{supp}\left\{C^{(1)}(\tau)\right\} \subset M^{-1}[-N, N]^{d}+M^{-1} \operatorname{supp} C .
$$

Thus

$$
\begin{aligned}
& \operatorname{supp}\left\{C^{(j)}(\tau)\right\} \subset M^{-1}[-N, N]^{d}+M^{-1} \operatorname{supp}\left\{C^{(j-1)}(\tau)\right\} \subset \cdots \\
& \quad \subset M^{-1}[-N, N]^{d}+\cdots+M^{-j}[-N, N]^{d}+M^{-j} \operatorname{supp} C \subset \Omega+M^{-j} \operatorname{supp} C .
\end{aligned}
$$


Since $\operatorname{dist}\left(\Omega, \mathbb{Z}^{d} \backslash[\Omega]\right)>0$ and $\lim _{j \rightarrow \infty} M^{-j}=0$, there exists $n \in \mathbb{Z}_{+}$such that

$$
\operatorname{dist}\left(\{0\}, M^{-n} \operatorname{supp} C\right)<\operatorname{dist}\left(\Omega, \mathbb{Z}^{d} \backslash[\Omega]\right) .
$$

Thus supp $\left\{C^{(n)}(\tau)\right\} \in[\Omega]$ and $\mathbf{T}^{n} C \in \mathbb{H}$.

Finally, if $C \in C_{0}\left(\mathbb{T}^{d}\right)$ is an eigenvector of $\mathbf{T}$ with corresponding eigenvalue $\lambda_{0} \neq 0$, then by (iii), $C=\lambda_{0}^{-1} \mathbf{T} C=\cdots=\lambda_{0}^{-n} \mathbf{T}^{n} C \in \mathbb{H}$.

Lemma 2.3. Let $\Phi$ be a compactly supported $(M, \mathbf{P})$ matrix refinable function and $G_{\Phi}$ be its Gram matrix defined by (1.6). If $G_{\Phi}(\omega)<\infty$ for all $\omega \in \mathbb{T}^{d}$, then

$$
\mathbf{T} G_{\Phi}=G_{\Phi}
$$

and if $\Phi \in L^{2}\left(\mathbb{R}^{d}\right)$, then $G_{\Phi} \in \mathbb{H}$.

Proof. By (1.2) and the definitions of $\mathbf{T}, G_{\Phi}$, we have

$$
\begin{aligned}
\mathbf{T} G_{\Phi}(\omega)= & \sum_{j=0}^{m-1} \sum_{\ell \in \mathbb{Z}^{d}} \mathbf{P}\left({ }^{t} M^{-1}\left(\omega+2 \pi \eta_{j}\right)\right) \widehat{\Phi}\left({ }^{t} M^{-1}\left(\omega+2 \pi \eta_{j}\right)+2 \pi \ell\right) \\
& . \widehat{\Phi}^{*}\left({ }^{t} M^{-1}\left(\omega+2 \pi \eta_{j}\right)+2 \pi \ell\right) \mathbf{P}^{*}\left({ }^{t} M^{-1}\left(\omega+2 \pi \eta_{j}\right)\right) \\
= & \sum_{j=0}^{m-1} \sum_{\ell \in \mathbb{Z}^{d}} \widehat{\Phi}\left(\omega+2 \pi \eta_{j}+2 \pi^{t} M \ell\right) \widehat{\Phi}^{*}\left(\omega+2 \pi \eta_{j}+2 \pi^{t} M \ell\right) \\
= & \sum_{\ell^{\prime} \in \mathbb{Z}^{d}} \widehat{\Phi}\left(\omega+2 \pi \ell^{\prime}\right) \widehat{\Phi}^{*}\left(\omega+2 \pi \ell^{\prime}\right)=G_{\Phi}(\omega) .
\end{aligned}
$$

By Lemma 2.2 and the Poisson summation formula, $G_{\Phi} \in \mathbb{H}$ if $\Phi \in L^{2}\left(\mathbb{R}^{d}\right)$.

In (2.6), the transition operator $\mathbf{T}$ is defined by (1.3) on the function space consisting of $r \times r$ matrix functions with every entry a $2 \pi$-periodic function.

We will show that if there is a compactly supported solution $\Phi$ of (1.1) satisfying $G_{\Phi}(\omega)<\infty$ and $\operatorname{det} G_{\Phi}(0) \neq 0$, then $\mathbf{P}(0)$ satisfies Condition E. For this, we first have

Proposition 2.4. Let $\Phi$ be a compactly supported matrix refinable function of (1.1) and let $\mathbf{1}$ be a left (row) eigenvector of an eigenvalue $\lambda_{0}$ of $\mathbf{P}(0)$ with $\left|\lambda_{0}\right| \geq 1$. If $G_{\Phi}(\omega)<\infty$, for $\omega \in \mathbb{T}^{d}$, then

$$
\mathbf{l} \widehat{\Phi}(2 \pi \beta)=0, \quad \beta \in \mathbb{Z}^{d} \backslash\{0\} .
$$

Proof. By (2.6),

$$
\begin{aligned}
& \mathbf{l} G_{\Phi}(0) \mathbf{l}^{*}=\mathbf{l} \mathbf{T} G_{\Phi}(0) \mathbf{l}^{*} \\
& =\left|\lambda_{0}\right|^{2} \mathbf{l} G_{\Phi}(0) \mathbf{l}^{*}+\sum_{j=1}^{m-1} \mathbf{l} \mathbf{P}\left(2 \pi^{t} M^{-1} \eta_{j}\right) G_{\Phi}\left(2 \pi^{t} M^{-1} \eta_{j}\right) \mathbf{P}^{*}\left(2 \pi^{t} M^{-1} \eta_{j}\right) \mathbf{l}^{*} \\
& \geq \mathbf{l} G_{\Phi}(0) \mathbf{l}^{*}+\sum_{j=1}^{m-1} \mathbf{l} \mathbf{P}\left(2 \pi^{t} M^{-1} \eta_{j}\right) G_{\Phi}\left(2 \pi^{t} M^{-1} \eta_{j}\right) \mathbf{P}^{*}\left(2 \pi^{t} M^{-1} \eta_{j}\right) \mathbf{l}^{*}
\end{aligned}
$$

Thus

$$
\sum_{j=1}^{m-1} \mathbf{l}\left(2 \pi^{t} M^{-1} \eta_{j}\right) G_{\Phi}\left(2 \pi^{t} M^{-1} \eta_{j}\right) \mathbf{P}^{*}\left(2 \pi^{t} M^{-1} \eta_{j}\right) \mathbf{1}^{*}=0
$$


By (1.2), we have

$$
\begin{aligned}
& \sum_{j=1}^{m-1} \sum_{\alpha \in \mathbb{Z}^{d}}\left|\widehat{\mathbf{\Phi}}\left(2 \pi \eta_{j}+2 \pi^{t} M \alpha\right)\right|^{2} \\
= & \sum_{j=1}^{m-1} \sum_{\alpha \in \mathbb{Z}^{d}} \mathbf{l} \mathbf{P}\left(2 \pi^{t} M^{-1} \eta_{j}\right) \widehat{\Phi}\left(2 \pi^{t} M^{-1} \eta_{j}+2 \pi \alpha\right) \\
& . \widehat{\Phi}\left(2 \pi^{t} M^{-1} \eta_{j}+2 \pi \alpha\right) \mathbf{P}\left(2 \pi^{t} M^{-1} \eta_{j}\right) \mathbf{l}^{*} \\
= & \sum_{j=1}^{m-1} \mathbf{l} \mathbf{P}\left(2 \pi^{t} M^{-1} \eta_{j}\right) G_{\Phi}\left(2 \pi^{t} M^{-1} \eta_{j}\right) \mathbf{P}^{*}\left(2 \pi^{t} M^{-1} \eta_{j}\right) \mathbf{l}^{*}=0 .
\end{aligned}
$$

Therefore,

$$
\mathbf{l} \widehat{\Phi}\left(2 \pi \eta_{j}+2 \pi^{t} M \alpha\right)=0, \quad 1 \leq j \leq m-1, \alpha \in \mathbb{Z}^{d} .
$$

For any $\beta \in \mathbb{Z}^{d} \backslash\{0\}$, there exist $j \in \mathbb{Z}_{+}, 1 \leq j \leq m-1, n \in \mathbb{Z}_{+}, \alpha \in \mathbb{Z}^{d}$ such that $\beta=\left({ }^{t} M\right)^{n}\left(\eta_{j}+{ }^{t} M \alpha\right)$. Thus

$$
\begin{aligned}
& \mathbf{l} \widehat{\Phi}(2 \pi \beta)=\mathbf{l} \mathbf{P}\left(2 \pi^{t} M^{-1} \beta\right) \cdots \mathbf{P}\left(2 \pi^{t} M^{-n} \beta\right) \widehat{\Phi}\left(2 \pi^{t} M^{-n} \beta\right) \\
& =\mathbf{l} \mathbf{P}(0)^{n} \widehat{\Phi}\left(2 \pi \eta_{j}+2 \pi^{t} M \alpha\right)=\lambda_{0}^{n} \mathbf{l} \widehat{\Phi}\left(2 \pi \eta_{j}+2 \pi^{t} M \alpha\right)=0 .
\end{aligned}
$$

This shows (2.7).

We note that if $\lambda_{0}$ is an eigenvalue of $\mathbf{P}(0)$ with $\left|\lambda_{0}\right| \geq 1$ and $\lambda_{0} \neq 1$, then for any left $\lambda_{0}$-eigenvector $\mathbf{l}$ of $\mathbf{P}(0), \mathbf{l} \widehat{\Phi}(2 \pi \beta)=0$ for all $\beta \in \mathbb{Z}^{d}$.

By Proposition 2.4, the following proposition can be obtained as in [21]. Its proof is presented here for the sake of completeness.

Proposition 2.5. Let $\Phi$ be a compactly supported $(M, \mathbf{P})$ refinable vector with $G_{\Phi}(\omega)<\infty$. If $\operatorname{det}\left(G_{\Phi}(0)\right) \neq 0$, then $\mathbf{P}(0)$ satisfies Condition $E$.

Proof. Let $\lambda_{0}$ be an eigenvalue of $\mathbf{P}(0)$ with $\left|\lambda_{0}\right| \geq 1$, and $\mathbf{l}$ be a corresponding left (row) eigenvector. If $\lambda_{0} \neq 1$, by Proposition $2.4, \mathbf{l} G_{\Phi}(0) \mathbf{l}^{*}=\mathbf{l} \widehat{\Phi}(0) \widehat{\Phi}^{*}(0) \mathbf{l}^{*}=0$. On the other hand, since $\Phi \neq 0$, the spectral radius of $\mathbf{P}(0) \geq 1$. These two facts imply that if $\operatorname{det}\left(G_{\Phi}(0)\right) \neq 0$, then 1 is the only eigenvalue of $\mathbf{P}(0)$ on the unit circle with $\widehat{\Phi}(0)$ being a corresponding right eigenvector, and all other eigenvalues are in the unit circle. If 1 is not simple, since $\widehat{\Phi}(0)$ is a right 1-eigenvector of $\mathbf{P}(0)$, then one can find a left (row) 1-eigenvector $\mathbf{l}$ of $\mathbf{P}(0)$ such that $\mathbf{l} \widehat{\Phi}(0)=0$, which again leads to $\mathbf{l} G_{\Phi}(0) \mathbf{l}^{*}=0$. Therefore, 1 has to be a simple eigenvalue of $\mathbf{P}(0)$, and hence $\mathbf{P}(0)$ satisfies Condition E.

Proposition 2.6. Assume that (1.1) has a compactly supported solution $\Phi$ with $G_{\Phi}(\omega)<\infty$. If $\operatorname{det}\left(G_{\Phi}\left(2 \pi^{t} M^{-1} \eta_{j}\right)\right) \neq 0, j=0, \cdots m-1$, then $\mathbf{P}(0)$ satisfies Condition $E$ and satisfies the vanishing moment conditions of order at least one, i.e.

$$
\mathbf{l P}\left(2 \pi^{t} M^{-1} \eta_{j}\right)=0, \quad 1 \leq j \leq m-1,
$$

where $\mathbf{1}$ is the left 1 -eigenvector of $\mathbf{P}(0)$. 
Proof. By Proposition 2.5, $\mathbf{P}(0)$ satisfies Condition E; and by (2.6),

$$
\begin{aligned}
& \mathbf{l} G_{\Phi}(0) \mathbf{l}^{*}=\mathbf{l} \mathbf{T} G_{\Phi}(0) \mathbf{l}^{*} \\
& =\mathbf{l} G_{\Phi}(0) \mathbf{l}^{*}+\sum_{j=1}^{m-1} \mathbf{l} \mathbf{P}\left(2 \pi^{t} M^{-1} \eta_{j}\right) G_{\Phi}\left(2 \pi^{t} M^{-1} \eta_{j}\right) \mathbf{P}^{*}\left(2 \pi^{t} M^{-1} \eta_{j}\right) \mathbf{l}^{*}
\end{aligned}
$$

Hence,

$$
\mathbf{l P}\left(2 \pi^{t} M^{-1} \eta_{j}\right) G_{\Phi}\left(2 \pi^{t} M^{-1} \eta_{j}\right)\left(\mathbf{l} \mathbf{P}\left(2 \pi^{t} M^{-1} \eta_{j}\right)\right)^{*}=0, \quad 1 \leq j \leq m-1 .
$$

Since $G_{\Phi}\left(2 \pi^{t} M^{-1} \eta_{j}\right)>0$, we have $\mathbf{l P}\left(2 \pi^{t} M^{-1} \eta_{j}\right)=0,1 \leq j \leq m-1$.

By Proposition 2.6, we have the following corollary.

Corollary 2.7. If (1.1) has a compactly supported solution $\Phi$ which is stable, then $\mathbf{P}(0)$ satisfies Condition $E$ and $\mathbf{P}$ satisfies the vanishing moment conditions of order one (2.8).

Here we note that the vanishing moment condition (2.8) is equivalent to

$$
\mathbf{l} \sum_{\alpha \in \mathbb{Z}^{d}} \mathbf{P}_{M \alpha+\gamma_{k}}=1, \quad 1 \leq k \leq m-1 .
$$

In fact if (2.9) holds, then for any $j \in \mathbb{Z}_{+}, 0 \leq j \leq m-1$, by (2.1)

$$
\begin{aligned}
& \mathbf{l}\left(2 \pi^{t} M^{-1} \eta_{j}\right)=\frac{1}{m} \mathbf{l} \sum_{\alpha \in \mathbb{Z}^{d}} \mathbf{P}_{\alpha} e^{-i 2 \pi^{t} \eta_{j} M^{-1} \alpha} \\
& =\frac{1}{m} \sum_{k=0}^{m-1} \mathbf{l} \sum_{\beta \in \mathbb{Z}^{d}} \mathbf{P}_{M \beta+\gamma_{k}} e^{-i 2 \pi^{t} \eta_{j} M^{-1}\left(M \beta+\gamma_{k}\right)} \\
& =\frac{1}{m} \sum_{k=0}^{m-1}\left(\mathbf{l} \sum_{\beta \in \mathbb{Z}^{d}} \mathbf{P}_{M \beta+\gamma_{k}}\right) e^{-i 2 \pi^{t} \eta_{j} M^{-1} \gamma_{k}} \\
& =\frac{1}{m} \sum_{k=0}^{m-1} e^{-i 2 \pi^{t} \eta_{j} M^{-1} \gamma_{k}}=\delta(j) .
\end{aligned}
$$

Conversely, if (2.8) holds, then for any $k \in \mathbb{Z}_{+}, 0 \leq k \leq m-1$, by (2.2)

$$
\begin{aligned}
1 & =\sum_{j=0}^{m-1} \mathbf{l}\left(2 \pi^{t} M^{-1} \eta_{j}\right) e^{i 2 \pi^{t} \eta_{j} M^{-1} \gamma_{k}} \\
& =\frac{1}{m} \sum_{j=0}^{m-1} \mathbf{l} \sum_{\beta \in \mathbb{Z}^{d}} \sum_{s=0}^{m-1} \mathbf{P}_{M \beta+\gamma_{s}} e^{-i 2 \pi^{t} \eta_{j} M^{-1} \gamma_{s}} e^{i 2 \pi^{t} \eta_{j} M^{-1} \gamma_{k}} \\
& =\frac{1}{m} \sum_{\beta \in \mathbb{Z}^{d}} \mathbf{l} \sum_{s=0}^{m-1} \mathbf{P}_{M \beta+\gamma_{s}} \sum_{j=0}^{m-1} e^{-i 2 \pi^{t} \eta_{j} M^{-1}\left(\gamma_{s}-\gamma_{k}\right)} \\
& =\frac{1}{m} \sum_{\beta \in \mathbb{Z}^{d}} \mathbf{l} \sum_{s=0}^{m-1} \mathbf{P}_{M \beta+\gamma_{s}} m \delta_{k}(s)=\mathbf{l} \sum_{\beta \in \mathbb{Z}^{d}} \mathbf{P}_{M \beta+\gamma_{k}},
\end{aligned}
$$

and therefore (2.9) holds. 
Corollary 2.8. If (1.1) has a compactly supported solution $\Phi$ which is stable, then $\mathbf{P}(0)$ satisfies Condition $E$ and $\mathbf{P}$ satisfies

$$
\mathbf{l} \sum_{\alpha \in \mathbb{Z}^{d}} \mathbf{P}_{M \alpha+\gamma_{k}}=1, \quad 1 \leq k \leq m-1
$$

where $\mathbf{l}$ is the left 1-eigenvector of $\mathbf{P}(0)$.

In the following we will assume that $\mathbf{P}(0)$ satisfies Condition $\mathrm{E}$ and let $\mathbf{r}$ be the unit right (column) 1-eigenvector of $\mathbf{P}(0)$. Let $\mathbf{l}$ be the left (row) 1-eigenvector of $\mathbf{P}(0)$ with $\mathbf{l r}=1$. Let $U$ be an $r \times r$ inverse matrix such that the first column of $U$ is $\mathbf{r}$ and $U^{-1} \mathbf{P}(0) U$ is the Jordan canonical form of $\mathbf{P}(0)$ with its (1,1)-entry 1 . Then ${ }^{t} \mathbf{e}_{1} U^{-1}$ is a left (row) 1-eigenvector of $\mathbf{P}(0)$ with ${ }^{t} \mathbf{e}_{1} U^{-1} \mathbf{r}={ }^{t} \mathbf{e}_{1} U^{-1} U \mathbf{e}_{1}=1$. Thus ${ }^{t} \mathbf{e}_{1} U^{-1}=1$.

Denote

$$
\Pi_{n}(\omega):=\chi_{[-\pi, \pi]^{d}}\left({ }^{t} M^{-n} \omega\right) \prod_{j=1}^{n} \mathbf{P}\left({ }^{t} M^{-j} \omega\right), \quad \Pi(\omega):=\prod_{j=1}^{\infty} \mathbf{P}\left({ }^{t} M^{-j} \omega\right) .
$$

Then, if $\mathbf{P}(0)$ satisfies Condition $\mathrm{E}, \Pi_{n}$ converges to $\Pi$ pointwise with

$$
\Pi(\omega) U=(\widehat{\Phi}(\omega), \mathbf{0}, \cdots, \mathbf{0})
$$

where

$$
\widehat{\Phi}(\omega):=\prod_{j=1}^{\infty} \mathbf{P}\left({ }^{t} M^{-j} \omega\right) \mathbf{r}
$$

and any other compactly supported solution $\Psi$ of $(1.1)$ with $\widehat{\Psi}(0) \neq 0$ is given by (2.11). About the convergence of the infinite product $\prod_{j=1}^{\infty} \mathbf{P}\left({ }^{t} M^{-j} \omega\right)$, see [3], [23] for $M=2 \mathbf{I}_{r}$, and [20] for general dilation matrices $M$.

By (2.10), we have, for any $r \times r$ matrix $A$,

$$
\begin{aligned}
& \Pi(\omega) A \Pi(\omega)^{*}=\Pi(\omega) U U^{-1} A\left(U^{-1}\right)^{*} U^{*} \Pi^{*}(\omega) \\
& =\widehat{\Phi}(\omega) \mathbf{e}_{1}^{T} U^{-1} A\left(U^{-1}\right)^{*} \mathbf{e}_{1} \widehat{\Phi}^{*}(\omega)=\left(\mathbf{l} A \mathbf{l}^{*}\right) \widehat{\Phi}(\omega) \widehat{\Phi}(\omega)^{*} .
\end{aligned}
$$

We will provide in the next proposition a characterization of the existence of $\mathrm{L}^{2}$ solutions of (1.1) under the assumption that $\mathbf{P}(0)$ satisfies Condition E. For this, we have the following lemma.

Lemma 2.9. For any $H_{1}(\omega), H_{2}(\omega) \in C_{0}\left(\mathbb{T}^{d}\right)$, and any positive integer $n$,

$$
\int_{\mathbb{T}^{d}} H_{1}(\omega)\left(\mathbf{T}^{n} H_{2}\right)(\omega) d \omega=\int_{\mathbb{R}^{d}} H_{1}(\omega) \Pi_{n}(\omega) H_{2}\left({ }^{t} M^{-n} \omega\right) \Pi_{n}^{*}(\omega) d \omega
$$


Proof. The proof of (2.12) can be carried out by induction. In fact for $n=1$,

$$
\begin{aligned}
& \int_{\mathbb{T}^{d}} H_{1}(\omega) \mathbf{T} H_{2}(\omega) d \omega=m \int_{\mathbb{R}^{d}} H_{1}\left({ }^{t} M \omega\right) \sum_{j=0}^{m-1} \mathbf{P}\left(\omega+2 \pi^{t} M^{-1} \eta_{j}\right) \\
& \cdot H_{2}\left(\omega+2 \pi^{t} M^{-1} \eta_{j}\right) \mathbf{P}^{*}\left(\omega+2 \pi^{t} M^{-1} \eta_{j}\right) \chi_{\mathbb{T}^{d}}\left({ }^{t} M \omega\right) d \omega \\
& =m \int_{\mathbb{R}^{d}} H_{1}\left({ }^{t} M \omega\right) \mathbf{P}(\omega) H_{2}(\omega) \mathbf{P}^{*}(\omega) \sum_{j=0}^{m-1} \chi_{\mathbb{T}^{d}}\left({ }^{t} M \omega-2 \pi \eta_{j}\right) d \omega \\
& =m \int_{\mathbb{T}^{d}} H_{1}\left({ }^{t} M \omega\right) \mathbf{P}(\omega) H_{2}(\omega) \mathbf{P}^{*}(\omega) \sum_{\beta \in \mathbb{Z}^{d}} \sum_{j=0}^{m-1} \chi_{\mathbb{T}^{d}}\left({ }^{t} M \omega-2 \pi^{t} M \beta-2 \pi \eta_{j}\right) d \omega \\
& =m \int_{\mathbb{T}^{d}} H_{1}\left({ }^{t} M \omega\right) \mathbf{P}(\omega) H_{2}(\omega) \mathbf{P}^{*}(\omega) d \omega \\
& =\int_{\mathbb{R}^{d}} H_{1}(\omega) \mathbf{P}\left({ }^{t} M^{-1} \omega\right) H_{2}\left({ }^{t} M^{-1} \omega\right) \mathbf{P}^{*}\left({ }^{t} M^{-1} \omega\right) \chi_{\mathbb{T}^{d}}\left({ }^{t} M^{-1} \omega\right) d \omega \\
& =\int_{\mathbb{R}^{d}} H_{1}(\omega) \Pi_{1}(\omega) H_{2}\left({ }^{t} M^{-1} \omega\right) \Pi_{1}^{*}(\omega) d \omega .
\end{aligned}
$$

For $n \in \mathbb{Z}_{+} \backslash\{0\}$, assume that (2.12) holds for any positive integers smaller than $n$; then

$$
\begin{aligned}
& \int_{\mathbb{T}^{d}} H_{1}(\omega)\left(\mathbf{T}^{n} H_{2}\right)(\omega) d \omega=\int_{\mathbb{R}^{d}} H_{1}(\omega) \Pi_{n-1}(\omega)\left(\mathbf{T} H_{2}\right)\left({ }^{t} M^{1-n} \omega\right) \Pi_{n-1}^{*}(\omega) d \omega \\
& =m^{n} \int_{\mathbb{R}^{d}} H_{1}\left({ }^{t} M^{n} \omega\right) \Pi_{n-1}\left({ }^{t} M^{n} \omega\right)\left(\mathbf{T} H_{2}\right)\left({ }^{t} M \omega\right) \Pi_{n-1}^{*}\left({ }^{t} M^{n} \omega\right) d \omega \\
& =m^{n} \int_{\mathbb{R}^{d}} H_{1}\left({ }^{t} M^{n} \omega\right) \Pi_{n-1}\left({ }^{t} M^{n} \omega\right) \sum_{j=0}^{m-1} \mathbf{P}\left(\omega+2 \pi^{t} M^{-1} \eta_{j}\right) H_{2}\left(\omega+2 \pi^{t} M^{-1} \eta_{j}\right) \\
& \quad \cdot \mathbf{P}^{*}\left(\omega+2 \pi^{t} M^{-1} \eta_{j}\right) \Pi_{n-1}^{*}\left({ }^{t} M^{n} \omega\right) \chi_{\mathbb{T}^{d}}\left({ }^{t} M \omega\right) d \omega \\
& =m^{n} \sum_{\beta \in \mathbb{Z}^{d}} \int_{\mathbb{T}^{d}} H_{1}\left({ }^{t} M^{n} \omega\right) \mathbf{P}\left({ }^{t} M^{n-1} \omega\right) \cdots \mathbf{P}\left({ }^{t} M \omega\right) \mathbf{P}(\omega) H_{2}(\omega) \\
& \quad \cdot \mathbf{P}^{*}(\omega) \cdots \mathbf{P}^{*}\left({ }^{t} M^{n-1} \omega\right) \sum_{j=0}^{m-1} \chi_{\mathbb{T}^{d}}\left({ }^{t} M \omega-2 \pi^{t} M \beta-2 \pi \eta_{j}\right) d \omega \\
& =m^{n} \int_{\mathbb{T}^{d}} H_{1}\left({ }^{t} M^{n} \omega\right) \mathbf{P}\left({ }^{t} M^{n-1} \omega\right) \cdots \mathbf{P}(\omega) H_{2}(\omega)\left(\mathbf{P}\left({ }^{t} M^{n-1} \omega\right) \cdots \mathbf{P}(\omega)\right)^{*} d \omega \\
& =\int_{\mathbb{R}^{d}} H_{1}(\omega) \Pi_{n}(\omega) H_{2}\left({ }^{t} M^{-n} \omega\right) \Pi_{n}^{*}(\omega) d \omega .
\end{aligned}
$$

Thus the proof by induction is completed.

Proposition 2.10. Suppose that $\mathbf{P}(0)$ satisfies Condition E. Then $\Phi$ defined by (2.11) is in $L^{2}\left(\mathbb{R}^{d}\right)$ if and only if there exists a positive semidefinite $H \in \mathbb{H}$ such that $\mathbf{T} H=H$ and $\mathbf{l} H(0) \mathbf{l}^{*}>0$.

Proof. Suppose $\Phi \in L^{2}\left(\mathbb{R}^{d}\right)$. Then the matrix $H(\omega):=G_{\Phi}(\omega) \in \mathbb{H}$, and $H(\omega) \geq \mathbf{0}$, $\mathbf{T} H=H$. By Proposition 2.4, $\mathbf{l} H(0) \mathbf{l}^{*}=\mathbf{l} \widehat{\mathbf{\Phi}}(0) \widehat{\Phi}^{*}(0) \mathbf{l}^{*}=|\mathbf{l} \mathbf{r}|^{2}=1$. 
Conversely, since the matrix $\Pi_{n}(\omega) H\left({ }^{t} M^{-n} \omega\right) \Pi_{n}^{*}(\omega)$ converges pointwise to the matrix

$$
\Pi(\omega) H(0) \Pi(\omega)^{*}=\left(\mathbf{l} H(0) \mathbf{l}^{*}\right) \widehat{\Phi}(\omega) \widehat{\Phi}(\omega)^{*},
$$

we have

$$
\begin{aligned}
& \left(\mathbf{l} H(0) \mathbf{l}^{*}\right) \int_{\mathbb{R}^{d}}|\widehat{\Phi}(\omega)|^{2} d \omega=\sum_{i=1}^{r} \int_{\mathbb{R}^{d}} \liminf _{n \rightarrow \infty}{ }^{t} \mathbf{e}_{i} \Pi_{n}(\omega) H\left({ }^{t} M^{-n} \omega\right) \Pi_{n}(\omega)^{*} \mathbf{e}_{i} d \omega \\
& \leq \sum_{i=1}^{r} \liminf _{n \rightarrow \infty} \int_{\mathbb{R}^{d}}{ }^{t} \mathbf{e}_{i} \Pi_{n}(\omega) H\left({ }^{t} M^{-n} \omega\right) \Pi_{n}(\omega)^{*} \mathbf{e}_{i} d \omega<\infty .
\end{aligned}
$$

The last inequality follows from the fact that

$$
\int_{\mathbb{R}^{d}} \Pi_{n}(\omega) H\left({ }^{t} M^{-n} \omega\right) \Pi_{n}^{*}(\omega) d \omega=\int_{\mathbb{T}^{d}}\left(\mathbf{T}^{n} H\right)(\omega) d \omega=\int_{\mathbb{T}^{d}} H(\omega) d \omega .
$$

About the existence of $L^{2}$-solutions of (1.1) for $M=2 \mathbf{I}_{r}$, a similar result was obtained in [21]. For the special case $r=1$ and $d=1$, this result was given in [28].

We will use the fact that if (1.1) has a compactly supported solution which is stable, then for any $H_{1}, H_{2} \in \mathbb{H}$,

$$
\lim _{n \rightarrow \infty} \int_{\mathbb{R}^{d}} \Pi_{n}(\omega) H_{1}\left({ }^{t} M^{-n} \omega\right) \Pi_{n}(\omega)^{*} H_{2}(\omega) d \omega=\int_{\mathbb{R}^{d}} \Pi(\omega) H_{1}(0) \Pi(\omega)^{*} H_{2}(\omega) d \omega .
$$

Equation (2.13) can be obtained as in [21] for the case $M=2 \mathbf{I}_{r}$, and we omit the details here.

The next theorem provides a characterization of the stability of the compactly supported $(M, \mathbf{P})$ refinable vector $\Phi$.

Theorem 2.11. The refinement equation (1.1) has a compactly supported solution which is stable if and only if the following conditions hold:

(i) the matrix $\mathbf{P}(0)$ satisfies Condition $E$,

(ii) for the left (row) 1-eigenvector $\mathbf{l}$ of $\mathbf{P}(0), \mathbf{P}\left(2 \pi^{t} M^{-1} \eta_{j}\right)=0,1 \leq j \leq m-1$,

(iii) the restriction transition operator $\mathbf{T}$ to $\mathbb{H}$ satisfies Condition E, and the corresponding 1-eigenvector is positive (or negative) definite on $\mathbb{T}^{d}$.

Proof. " $\Leftarrow$ " Let $H_{0} \in \mathbb{H}$ be the positive definite 1-eigenvector of T. By Proposition 2.10, the solution $\Phi$ given by (2.11) is in $L^{2}\left(\mathbb{R}^{d}\right)$. Let $H(\omega)=G_{\Phi}(\omega)$; then $H(\omega) \in$ $\mathbb{H}$ and $\mathbf{T} H=H$. Since the restriction $\left.\mathbf{T}\right|_{\mathbb{H}}$ of $\mathbf{T}$ to $\mathbb{H}$ satisfies Condition $\mathrm{E}, H=c H_{0}$ for some positive constant $c$. Thus $G_{\Phi}(\omega)=c H_{0}(\omega)>0$, and hence $\Phi$ is stable.

" $\Rightarrow$ " Let $\Phi$ be a compactly supported solution which is stable; then $\widehat{\Phi}(0)=c \mathbf{r}$ for some nonzero constant $c$. (i), (ii) follow from Proposition 2.6. To complete the proof of Theorem 2.11, it is enough to show that the restricted operator $\left.\mathbf{T}\right|_{\mathbb{H}}$ satisfies Condition $\mathrm{E}$, since $G_{\Phi}$ is a positive definite 1-eigenvector of $\left.\mathbf{T}\right|_{\mathbb{H}}$.

Let $\lambda_{0}$ be an eigenvalue of $\left.\mathbf{T}\right|_{\mathbb{H}}$ and $H$ be a corresponding eigenvector. Since

$$
\begin{aligned}
& \lambda_{0}^{n} \int_{\mathbb{T}^{d}} H(\omega) H(\omega)^{*} d \omega=\int_{\mathbb{T}^{d}} \mathbf{T}^{n} H(\omega) H(\omega)^{*} d \omega \\
& =\int_{\mathbb{R}^{d}} \Pi_{n}(\omega) H\left({ }^{t} M^{-n} \omega\right) \Pi_{n}(\omega)^{*} H(\omega)^{*} d \omega,
\end{aligned}
$$


the $\operatorname{limit}_{\lim _{n \rightarrow \infty}} \lambda_{0}^{n}$ exists. Thus $\left|\lambda_{0}\right| \leq 1$, and 1 is the only eigenvalue of $\left.\mathbf{T}\right|_{\mathbb{H}}$ on the unit circle.

For an eigenvector $H$ of eigenvalue 1 of $\left.\mathbf{T}\right|_{\mathbb{H}}$, denote $c_{0}=\mathbf{l} H(0) \mathbf{l}^{*}$. Then

$$
\begin{aligned}
& \int_{\mathbb{T}^{d}}\left(H-c_{0} G_{\Phi}\right)\left(H-c_{0} G_{\Phi}\right)^{*} d \omega \\
& =\int_{\mathbb{R}^{d}} \Pi_{n}(\omega)\left(H\left({ }^{t} M^{-n} \omega\right)-c_{0} G_{\Phi}\left({ }^{t} M^{-n} \omega\right)\right) \Pi_{n}(\omega)^{*}\left(H(\omega)-c_{0} G_{\Phi}(\omega)\right)^{*} d \omega \\
& \rightarrow \int_{\mathbb{R}^{d}} \Pi(\omega)\left(H(0)-c_{0} G_{\Phi}(0)\right) \Pi(\omega)^{*}\left(H(\omega)-c_{0} G_{\Phi}(\omega)\right)^{*} d \omega \\
& =\mathbf{l}\left(H(0)-c_{0} G_{\Phi}(0)\right) \mathbf{l}^{*} \int_{\mathbb{R}^{d}} \widehat{\Phi}(\omega) \widehat{\Phi}^{*}(\omega)\left(H(\omega)-c_{0} G_{\Phi}(\omega)\right)^{*} d \omega=0 .
\end{aligned}
$$

Thus $H(\omega)=c_{0} G_{\Phi}(\omega)$. This implies that the geometric multiplicity of the eigenvalue 1 of $\left.\mathbf{T}\right|_{\mathbb{H}}$ is 1 .

Finally we show that 1 is nondegenerate. Otherwise, there exists $H \in \mathbb{H}$ such that $\mathbf{T} H=G_{\Phi}+H$. Let $H_{1}=H-c_{1} G_{\Phi}$, where $c_{1}=\mathbf{l} H(0) \mathbf{l}^{*}$. Then

$$
\begin{aligned}
& \int_{\mathbb{T}^{d}} \mathbf{T}^{n} H_{1}(\omega) G_{\Phi}(\omega)^{*} d \omega=\int_{\mathbb{R}^{d}} \Pi_{n}(\omega) H_{1}\left({ }^{t} M^{-n} \omega\right) \Pi_{n}(\omega)^{*} G_{\Phi}(\omega)^{*} d \omega \\
& \rightarrow \int_{\mathbb{R}^{d}} \Pi(\omega)\left(H(0)-c_{1} G_{\Phi}(0)\right) \Pi(\omega)^{*} G_{\Phi}(\omega)^{*} d \omega=0 .
\end{aligned}
$$

On the other hand,

$$
\mathbf{T}^{n} H_{1}=\mathbf{T}^{n} H-c_{1} G_{\Phi}=n G_{\Phi}+H-c_{1} G_{\Phi} ;
$$

thus $\left\|\int_{\mathbb{T}^{d}} \mathbf{T}^{n} H_{1}(\omega) G_{\Phi}(\omega)^{*} d \omega\right\| \rightarrow \infty$ as $n \rightarrow \infty$. This leads to a contradiction

The next theorem provides a characterization of the orthonormality of the compactly supported $(M, \mathbf{P})$ refinable vector $\Phi$.

Theorem 2.12. The refinement equation (1.1) has a compactly supported solution which is orthogonal if and only if the following conditions hold:

(i) the mask $\mathbf{P}$ is a $C Q F$,

(ii) the matrix $\mathbf{P}(0)$ satisfies Condition $E$,

(iii) for the left (row) 1-eigenvector $\mathbf{l}$ of $\mathbf{P}(0), \mathbf{l} \mathbf{P}\left(2 \pi^{t} M^{-1} \eta_{j}\right)=0,1 \leq j \leq m-1$,

(iv) the restriction of the transition operator $\mathbf{T}$ to $\mathbb{H}$ satisfies Condition $E$.

Proof. " $\Leftarrow$ " Since $\mathbf{P}$ is a CQF, $\mathbf{T I}_{r}=\mathbf{I}_{r}$. Therefore by Proposition 2.10, the compactly supported solution $\Phi$ given by $(2.11)$ is in $L^{2}\left(\mathbb{R}^{d}\right)$. By (iv), $G_{\Phi}=c \mathbf{I}_{r}$ for some positive constant $c$, and hence (1.1) has a compactly supported solution which is orthogonal.

" $\Rightarrow$ " (ii), (iii) and (iv) follow from the orthonormality of $\Phi$ and Theorem 2.11. By the orthonormality of $\Phi, G_{\Phi}(\omega)=\mathbf{I}_{r}$. Thus $\mathbf{T I}_{r}=\mathbf{I}_{r}$, i.e.

$$
\sum_{j=0}^{m-1} \mathbf{P}\left({ }^{t} M^{-1}\left(\omega+2 \pi \eta_{j}\right)\right) \mathbf{P}^{*}\left({ }^{t} M^{-1}\left(\omega+2 \pi \eta_{j}\right)\right)=\mathbf{I}_{r},
$$

and hence $\mathbf{P}$ is a CQF. 


\section{Approximation ORDER}

In this section we will consider the approximation order of the matrix refinable function $\Phi$. Throughout this section, we will assume the eigenvalues of the dilation matrix $M$ are nondegenerate.

Let ${ }^{t} M$ be the transpose of $M$ and $\lambda_{j}, j=1, \cdots, r$, be the eigenvalues of $M$. By our assumptions, $\left|\lambda_{i}\right|>1$ and every $\lambda_{i}$ is nondegenerate. Thus, there exist $d$ linearly independent vectors $\mathbf{v}^{1}, \cdots, \mathbf{v}^{d}$ such that ${ }^{t} M \mathbf{v}^{j}=\lambda_{j} \mathbf{v}^{j}, j=1, \ldots, d$. Let

$$
V:=\left(\mathbf{v}^{1}, \mathbf{v}^{2}, \ldots, \mathbf{v}^{d}\right)
$$

be the $d \times d$ matrix with column vectors $\mathbf{v}^{1}, \ldots, \mathbf{v}^{d}$. Then

$$
{ }^{t} M V=\left(\lambda_{1} \mathbf{v}^{1}, \cdots, \lambda_{d} \mathbf{v}^{d}\right)=V \Lambda,
$$

where $\Lambda:=\operatorname{diag}\left(\lambda_{1}, \ldots, \lambda_{d}\right)$. Denote

$$
\lambda:={ }^{t}\left(\lambda_{1}, \cdots, \lambda_{d}\right)
$$

Then for any $x \in \mathbb{R}^{d}, \beta \in \mathbb{Z}_{+}^{d}$,

$$
(\Lambda x)^{\beta}=\lambda^{\beta} x^{\beta} .
$$

For $1 \leq j \leq d$, let $D_{\mathbf{v}^{j}}$ denote the derivative operator in the direction $\mathbf{v}^{j}$, i.e.

$$
D_{\mathbf{v}^{j}}:=\left(\partial_{1}, \cdots, \partial_{d}\right) \mathbf{v}^{j}
$$

Then

$$
D_{\mathbf{v}^{j}} f\left({ }^{t} M \omega\right)=\lambda_{j}\left(D_{\mathbf{v}^{j}} f\right)\left({ }^{t} M \omega\right)
$$

For $\beta={ }^{t}\left(\beta_{1}, \cdots, \beta\right) \in \mathbb{Z}_{+}^{d}$, denote

$$
D_{V}^{\beta}:=D_{\mathbf{v}^{1}}^{\beta_{1}} \cdots D_{\mathbf{v}^{d}}^{\beta_{d}}
$$

Then we have

$$
D_{V}^{\beta} f\left({ }^{t} M \omega\right)=\lambda^{\beta}\left(D_{V}^{\beta} f\right)\left({ }^{t} M \omega\right), \quad \beta \in \mathbb{Z}_{+}^{d} .
$$

For a compactly supported vector-valued function $\Psi={ }^{t}\left(\psi_{1}, \cdots, \psi_{r}\right)$, we denote by $\mathcal{S}(\Psi)$ the linear space of all functions of the form $\sum_{i=1}^{r} \sum_{\ell \in \mathbb{Z}^{d}} c_{i}(\ell) \psi_{i}(\cdot-\ell)$, where $\left\{c_{i}(\ell)\right\}_{\ell \in \mathbb{Z}^{d}}$ are arbitrary sequences on $\mathbb{Z}^{d}$.

We say $\Psi$ has accuracy of order $k$ if all polynomials of total degree smaller than $k$ are contained in $\mathcal{S}(\Psi)$, i.e. for any $\beta \in \mathbb{Z}_{+}^{d},|\beta|<k$, there exist $y_{\beta, i}(\ell)$ such that

$$
x^{\beta}=\sum_{i=1}^{r} \sum_{\ell \in \mathbb{Z}^{d}} y_{\beta, i}(\ell) \psi_{i}(x+\ell) .
$$

For $\Psi \in L^{2}\left(\mathbb{R}^{d}\right)$ and $h>0$, let

$$
S_{h}(\Psi):=\left\{f\left(\frac{\dot{h}}{h}\right): \quad f \in \mathcal{S}(\Psi) \cap L^{2}\left(\mathbb{R}^{d}\right)\right\}
$$

be the $h$-dilation of $\mathcal{S}(\Psi) \cap L^{2}\left(\mathbb{R}^{d}\right)$. For $k>0$, we say $\Psi$ (or $\mathcal{S}(\Psi)$ ) provides $L^{2}$ approximation of order $k$ if for every sufficiently smooth function $f \in L^{2}\left(\mathbb{R}^{d}\right)$ and any $h>0$

$$
\operatorname{dist}\left(f, S_{h}(\Psi)\right)=O\left(h^{k}\right)
$$

where dist here is the $L^{2}$-distance between a function and a subset of $L^{2}\left(\mathbb{R}^{d}\right)$. 
An $r \times 1$ vector-valued function $\Psi$ is said to satisfy the Strang-Fix conditions of order $k$ if there is a finitely supported $1 \times r$ vector-valued sequence $\left\{q_{\ell}\right\}_{\ell \in \mathbb{Z}^{d}}$ such that $f:=\sum_{\ell \in \mathbb{Z}^{d}} q_{\ell} \Psi(\cdot-\ell)$ satisfies

$$
D^{\beta} \widehat{f}(2 \pi \ell)=\delta(\beta) \delta(\ell), \quad \text { for } \ell \in \mathbb{Z}^{d}, \beta \in \mathbb{Z}_{+}^{d},|\beta|<k .
$$

About the relations among the orders of accuracy, $L^{2}$-approximation and StrangFix conditions of $\Psi$, see [13] and the references therein. The next theorem was obtained by Jia (see [13], [14]).

Theorem 3.1. (Jia). Let $\Psi={ }^{t}\left(\psi_{1}, \cdots, \psi_{r}\right) \in L^{2}\left(\mathbb{R}^{d}\right)$ be a compactly supported vector-valued function. Assume that the sequences $\left(\widehat{\psi}_{j}(2 \pi \beta)\right)_{\beta \in \mathbb{Z}^{d}}, j=1, \cdots, r$, are linearly independent. Then the following statements are equivalent:

(a) $\Psi$ provides $L_{2}$-approximation of order $k$;

(b) $\Psi$ has accuracy of order $k$;

(c) $\Psi$ satisfies the Strang-Fix conditions of order $k$.

For a compactly supported $(M, \mathbf{P})$ refinable vector $\Phi$, we will find the $L^{2}$ approximation order of $\Phi$ in terms of the mask $\mathbf{P}$. For a given mask $\mathbf{P}$, if there exist a positive integer $k$ and $1 \times r$ complex vectors $\mathbf{l}_{0}^{\beta},|\beta|<k$, with $\mathbf{l}_{0}^{0} \neq 0$, such that

$$
\sum_{0 \leq \alpha \leq \beta}\left(\begin{array}{c}
\beta \\
\alpha
\end{array}\right)(i \lambda)^{\alpha-\beta} \mathbf{l}_{0}^{\alpha} D_{V}^{\beta-\alpha} \mathbf{P}\left(2 \pi^{t} M^{-1} \eta_{j}\right)=\delta(j) \lambda^{-\beta} \mathbf{l}_{0}^{\beta}, \quad 0 \leq j \leq m-1,
$$

we say that the refinement mask $\mathbf{P}$ satisfies the vanishing moment conditions of order $k$.

We show in the next theorem that if $\mathbf{P}$ satisfies the vanishing moment conditions of order $k$ and $\Phi \in L^{2}\left(\mathbb{R}^{d}\right)$ is a compactly supported $(M, \mathbf{P})$ refinable vector with $\mathbf{l}_{0}^{0} \widehat{\Phi}(0) \neq 0$, then $\Phi$ satisfies the Strang-Fix conditions of order $k$.

Theorem 3.2. If $\mathbf{P}$ satisfies the vanishing moment conditions of order $k$, i.e. there exist $1 \times r$ complex vectors $\mathbf{l}_{0}^{\beta},|\beta|<k$, with $\mathbf{l}_{0}^{0} \neq 0$ such that (3.4) holds, then any compactly supported $(\mathbf{P}, M)$ refinable vector $\Phi \in L^{2}(\mathbb{R})$ with $\mathbf{l}_{0}^{0} \widehat{\Phi}(0) \neq 0$ satisfies the Strang-Fix conditions of order $k$.

Proof. Let $f$ be the vector-valued function in $L^{2}\left(\mathbb{R}^{d}\right)$ defined by

$$
\widehat{f}(\omega):=b(\omega) \widehat{\Phi}(\omega)
$$

where $b(\omega)$ is the vector-valued function given by $b(\omega)=\sum_{|\ell|<k} b_{\ell} e^{i \ell \omega}$ with

$$
(-i)^{|\beta|} D_{V}^{\beta} b(0)=\sum_{|\ell|<k}\left({ }^{t} V \ell\right)^{\beta} b_{\ell}=\mathbf{l}_{0}^{\beta}, \quad|\beta|<k .
$$

We will show that $f$ satisfies the Strang-Fix conditions of order $k$.

Since $\left(\partial_{1}, \cdots, \partial_{d}\right)=\left(D_{v^{1}}, \cdots, D_{v^{d}}\right) V^{-1}$, it is enough to show that

$$
D_{V}^{\beta} \widehat{f}(2 \pi \ell)=c \delta(\beta) \delta(\ell), \quad \text { for } \ell \in \mathbb{Z}^{d} \text { and } \beta \in \mathbb{Z}_{+}^{d},|\beta|<k,
$$

where $c$ is a nonzero constant.

One can check that (3.4) is equivalent to

$\left.D_{V}^{\beta}\left(b(\omega) \mathbf{P}\left({ }^{t} M^{-1} \omega\right)\right)\right|_{\omega=2 \pi \eta_{j}}=\delta(j) \lambda^{-\beta} D_{V}^{\beta} b(0), \quad 0 \leq j \leq m-1, \beta \in \mathbb{Z}_{+}^{d},|\beta|<k$. 
For any $\ell \in \mathbb{Z}^{d}$, there exists $j, 0 \leq j \leq m-1$, such that $\ell \in \eta_{j}+{ }^{t} M \mathbb{Z}^{d}$. By (3.2), one has

$$
\begin{aligned}
& D_{V}^{\beta} \widehat{f}(2 \pi \ell)=\left.D_{V}^{\beta}\left(b(\omega) \mathbf{P}\left({ }^{t} M^{-1} \omega\right) \widehat{\Phi}\left({ }^{t} M^{-1} \omega\right)\right)\right|_{\omega=2 \pi \ell} \\
& =\left.\left.\sum_{0 \leq \alpha \leq \beta}\left(\begin{array}{c}
\beta \\
\alpha
\end{array}\right) D_{V}^{\alpha}\left(b(\omega) \mathbf{P}\left({ }^{t} M^{-1} \omega\right)\right)\right|_{\omega=2 \pi \ell} D_{V}^{\beta-\alpha}\left(\widehat{\Phi}\left({ }^{t} M^{-1} \omega\right)\right)\right|_{\omega=2 \pi \ell} \\
& =\left.\sum_{0 \leq \alpha \leq \beta}\left(\begin{array}{c}
\beta \\
\alpha
\end{array}\right) D_{V}^{\alpha}\left(b(\omega) \mathbf{P}\left({ }^{t} M^{-1} \omega\right)\right)\right|_{\omega=2 \pi \eta_{j}} \lambda^{\alpha-\beta} D_{V}^{\beta-\alpha} \widehat{\Phi}\left(2 \pi^{t} M^{-1} \ell\right) \\
& =\sum_{0 \leq \alpha \leq \beta}\left(\begin{array}{c}
\beta \\
\alpha
\end{array}\right) \lambda^{-\alpha} D_{V}^{\alpha} b(0) \delta(j) \lambda^{\alpha-\beta} D_{V}^{\beta-\alpha} \widehat{\Phi}\left(2 \pi^{t} M^{-1} \ell\right) \\
& =\delta(j) \lambda^{-\beta} \sum_{0 \leq \alpha \leq \beta}\left(\begin{array}{c}
\beta \\
\alpha
\end{array}\right) D_{V}^{\alpha} b\left(2 \pi^{t} M^{-1} \ell\right) D_{V}^{\beta-\alpha} \widehat{\Phi}\left(2 \pi^{t} M^{-1} \ell\right) \\
& =\delta(j) \lambda^{-\beta} D_{V}^{\beta} \widehat{f}\left(2 \pi^{t} M^{-1} \ell\right) ;
\end{aligned}
$$

the next to last equality is because if $j=0$, then $D_{V}^{\alpha} b\left(2 \pi^{t} M^{-1} \ell\right)=D_{V}^{\alpha} b(0)$ by $2 \pi$-periodicity of $b(\omega)$, and if $j \neq 0$, both sides are zero. So we have

$$
D_{V}^{\beta} \widehat{f}(2 \pi \ell)=\delta(j) \lambda^{-\beta} D_{V}^{\beta} \widehat{f}\left(2 \pi^{t} M^{-1} \ell\right), \quad \ell \in \eta_{j}+{ }^{t} M \mathbb{Z}^{d} .
$$

If $\ell \neq 0$, by repeating this procedure, we have $D_{V}^{\beta} \widehat{f}(2 \pi \ell)=0$. And if $\ell=0, \beta \neq 0$, then by (3.8), $D_{V}^{\beta} \widehat{f}(0)=\lambda^{-\beta} D_{V}^{\beta} \widehat{f}(0)$. Thus $D_{V}^{\beta} \widehat{f}(0)=0$ since $\lambda^{-\beta} \neq 1$. Finally, if $\ell=0, \beta=0$, then

$$
\widehat{f}(0)=b(0) \widehat{\Phi}(0)=\mathbf{l}_{0}^{0} \widehat{\Phi}(0) \neq 0 .
$$

Therefore we have (3.7) with $c=\mathbf{l}_{0}^{0} \widehat{\Phi}(0)$, and proved Theorem 3.2.

Remark 3.3. We note that $\mathbf{l}_{0}^{0}$ in (3.4) is a left 1-eigenvector of $\mathbf{P}(0)$. Thus if $\mathbf{P}(0)$ satisfies Condition E, then the solution $\Phi \in L^{2}\left(\mathbb{R}^{d}\right)$ of $(1.1)$ with $\mathbf{l}_{0}^{0} \widehat{\Phi}(0) \neq 0$ is given by (2.11), and $\Phi$ given by (2.11) satisfies $\mathbf{l}_{0}^{0} \widehat{\Phi}(0) \neq 0$.

Remark 3.4. Note that for a compactly supported vector-valued function $\Psi \in$ $L^{2}\left(\mathbb{R}^{2}\right)$, the condition that $\left(\widehat{\psi}_{j}(2 \pi \beta)\right)_{\beta \in \mathbb{Z}^{d}}, j=1, \cdots, r$, are linearly independent in Theorem 3.1 (Jia) is equivalent to $\operatorname{det}\left(G_{\Phi}(0)\right) \neq 0$. Theorem 4.2 in [7] says that under the mild condition $\operatorname{det}\left(G_{\Phi}(0)\right) \neq 0, \Phi$ providing $L^{2}$-approximation of order $k$ implies that the finitely supported $1 \times r$ vector-valued sequence $\left\{q_{\ell}\right\}_{\ell \in \mathbb{Z}^{d}}$ with $f:=\sum_{\ell \in \mathbb{Z}^{d}} q_{\ell} \Phi(\cdot-\ell)$ satisfying $(3.3)$ is unique.

The above two remarks lead to the following proposition about the uniqueness of the vectors $\mathbf{l}_{0}^{\beta}$ satisfying (3.4).

Proposition 3.5. Assume that $\mathbf{P}$ satisfies the vanishing moment conditions of order $k$ with vectors $\mathbf{l}_{0}^{\beta}, \beta \in \mathbb{Z}_{+}^{d},|\beta|<k, \mathbf{l}_{0}^{0} \neq 0$ satisfying (3.4). If (1.1) has a compactly supported solution $\Phi \in L^{2}\left(\mathbb{R}^{d}\right)$ satisfying $\operatorname{det}\left(G_{\Phi}(0)\right) \neq 0$, then, up to a constant, the vectors $\mathbf{1}_{0}^{\beta}, \beta \in \mathbb{Z}_{+}^{d},|\beta|<k$, are unique.

Proof. Assume that $\mathbf{l}_{0}^{\beta}, \beta \in \mathbb{Z}_{+}^{d},|\beta|<k, \mathbf{l}_{0}^{0} \neq 0$ are vectors satisfying (3.4). Since $\operatorname{det}\left(G_{\Phi}(0)\right) \neq 0, \mathbf{P}(0)$ satisfies Condition $\mathrm{E}$ with $\widehat{\Phi}(0)$ being a right 1-eigenvector of $\mathbf{P}(0)$. Hence $\mathbf{l}_{0}^{0} \widehat{\Phi}(0) \neq 0$. Let $f$ be the function defined by (3.5) with $\left\{b_{\ell}\right\}$ defined by (3.6). As shown in the proof of Theorem 3.2, $f$ satisfies (3.3). Since $\operatorname{det}\left(G_{\Phi}(0)\right) \neq 0$, 
by Theorem 4.2 in [7], the sequence $\left\{b_{\ell}\right\}$ is unique (up to a constant). Hence the vectors $\mathbf{l}_{0}^{\beta}$ are also unique.

The next theorem will show that, under mild conditions, $\mathbf{P}$ satisfying the vanishing moment conditions of order $k$ is also necessary for $\Phi$ to provide $L^{2}$-approximation of order $k$.

Theorem 3.6. Assume that $\Phi \in L^{2}\left(\mathbb{R}^{d}\right)$ is a compactly supported $(M, \mathbf{P})$ refinable vector and $\operatorname{det}\left(G_{\Phi}\left(2 \pi^{t} M^{-1} \eta_{j}\right)\right) \neq 0, j=0, \cdots, m-1$. Then the following conditions are equivalent:

(i) $\Phi$ provides approximation of order $k$;

(ii) $\Phi$ has accuracy of order $k$;

(iii) $\Phi$ satisfies the Strang-Fix conditions of order $k$;

(iv) the matrix refinement mask $\mathbf{P}$ satisfies the vanishing moment conditions of order $k$.

Proof. The equivalence of (i), (ii) and (iii) is proved in Theorem 3.1 (Jia). Since $\operatorname{det}\left(G_{\Phi}(0)\right) \neq 0$, by Proposition 2.5, $\mathbf{P}(0)$ satisfies Condition E. Thus by Remark 3.3 and Theorem 3.2 , we know that (iv) $\Rightarrow$ (iii), and we need only to show that (iii) $\Rightarrow$ (iv).

Let $\left\{q_{\ell}\right\}$ be the finitely supported $1 \times r$ vector-valued sequence such that $f=$ $\sum_{\ell \in \mathbb{Z}^{d}} q_{\ell} \Phi(\cdot-\ell)$ satisfies (3.7) with $c=1$. Let $\widehat{q}(\omega)$ denote the Fourier series of $\left\{q_{\ell}\right\}$; then $\widehat{f}(\omega)=\widehat{q}(\omega) \widehat{\Phi}(\omega)$. We will prove by induction that

$\left.D_{V}^{\beta}\left(\widehat{q}(\omega) \mathbf{P}\left({ }^{t} M^{-1} \omega\right)\right)\right|_{\omega=2 \pi \eta_{j}}=\delta(j) \lambda^{-\beta} D_{V}^{\beta} \widehat{q}(0), \quad 0 \leq j \leq m-1, \beta \in \mathbb{Z}_{+}^{d},|\beta|<k$, which is equivalent to (3.4) with $\mathbf{l}_{0}^{\beta}=(-i)^{|\beta|} D_{V}^{\beta} \widehat{q}(0)$. $\mathbb{Z}^{d}$,

First we have $\widehat{f}(0)=\widehat{q}(0) \widehat{\Phi}(0) \neq 0$; thus $\mathbf{l}_{0}^{0}=\widehat{q}(0) \neq 0$. Since $\widehat{f}(2 \pi \kappa)=\delta(\kappa), \kappa \in$

$$
\widehat{q}(0) \mathbf{P}\left(2 \pi^{t} M^{-1} \kappa\right) \widehat{\Phi}\left(2 \pi^{t} M^{-1} \kappa\right)=\delta(\kappa) .
$$

Hence for any $j \in \mathbb{Z}_{+}, 0 \leq j \leq m-1$, and $\ell \in \mathbb{Z}^{d}$,

$$
\widehat{q}(0) \mathbf{P}\left(2 \pi^{t} M^{-1} \eta_{j}\right) \widehat{\Phi}\left(2 \pi \ell+2 \pi^{t} M^{-1} \eta_{j}\right)=\delta(\ell) \delta(j) .
$$

Multiplying both sides of (3.10) by $\widehat{\Phi}^{*}\left(2 \pi \ell+2 \pi^{t} M^{-1} \eta_{j}\right)$ and summing over $\ell \in \mathbb{Z}^{d}$,

$$
\widehat{q}(0) \mathbf{P}\left(2 \pi^{t} M^{-1} \eta_{j}\right) G_{\Phi}\left(2 \pi^{t} M^{-1} \eta_{j}\right)=\delta(j) \widehat{\Phi}^{*}(0)
$$

If $j \neq 0$, then by the invertibility of $G_{\Phi}\left(2 \pi^{t} M^{-1} \eta_{j}\right)$, we have $\widehat{q}(0) \mathbf{P}\left(2 \pi^{t} M^{-1} \eta_{j}\right)=0$, and if $j=0$, then we have

$$
\widehat{q}(0) \mathbf{P}(0)=\widehat{\Phi}^{*}(0) G_{\Phi}(0)^{-1}
$$

On the other hand, since $\widehat{f}(2 \pi \kappa)=\delta(\kappa), \kappa \in \mathbb{Z}^{d}$, we have $\widehat{q}(0) \widehat{\Phi}(2 \pi \kappa)=\delta(\kappa)$. This again leads to $\widehat{q}(0) G_{\Phi}(0)=\widehat{\Phi}^{*}(0)$, i.e. $\widehat{q}(0)=\widehat{\Phi}^{*}(0) G_{\Phi}(0)^{-1}$. Therefore we have $\widehat{q}(0) \mathbf{P}(0)=\widehat{q}(0)$, and (3.9) is true for $\beta=0$.

For $\beta \in \mathbb{Z}_{+}^{d} \backslash\{0\},|\beta|<k$, assume that (3.9) is true any $\alpha<\beta, \alpha \in \mathbb{Z}_{+}^{d}$. We want to prove that (3.9) holds for $\beta$.

Since $D_{V}^{\beta} \widehat{f}(2 \pi \kappa)=0$, for all $\kappa \in \mathbb{Z}^{d}$

$$
\left.\left.\sum_{0 \leq \alpha \leq \beta}\left(\begin{array}{c}
\beta \\
\alpha
\end{array}\right) D_{V}^{\alpha}\left(\widehat{q}(\omega) \mathbf{P}\left({ }^{t} M^{-1} \omega\right)\right)\right|_{\omega=2 \pi \kappa} D_{V}^{\beta-\alpha}\left(\widehat{\Phi}\left({ }^{t} M^{-1} \omega\right)\right)\right|_{\omega=2 \pi \kappa}=0
$$


and hence for any $j \in \mathbb{Z}_{+}, 0 \leq j \leq m-1$, and $\ell \in \mathbb{Z}^{d}$

$$
\left.\left.\sum_{0 \leq \alpha \leq \beta}\left(\begin{array}{c}
\beta \\
\alpha
\end{array}\right) D_{V}^{\alpha}\left(\widehat{q}(\omega) \mathbf{P}\left({ }^{t} M^{-1} \omega\right)\right)\right|_{\omega=2 \pi \eta_{j}} D_{V}^{\beta-\alpha}\left(\widehat{\Phi}\left({ }^{t} M^{-1} \omega\right)\right)\right|_{\omega=2 \pi^{t} M \ell+2 \pi \eta_{j}}=0 .
$$

By (3.9) for $\alpha<\beta$,

$$
\begin{aligned}
& \left.D_{V}^{\beta}\left(\widehat{q}(\omega) \mathbf{P}\left({ }^{t} M^{-1} \omega\right)\right)\right|_{\omega=2 \pi \eta_{j}} \widehat{\Phi}\left(2 \pi \ell+2 \pi^{t} M^{-1} \eta_{j}\right) \\
& \quad=-\sum_{0 \leq \alpha<\beta}\left(\begin{array}{c}
\beta \\
\alpha
\end{array}\right) \lambda^{-\alpha} \delta(j) D_{V}^{\alpha} \widehat{q}(0) \lambda^{\alpha-\beta} D_{V}^{\beta-\alpha} \widehat{\Phi}\left(2 \pi \ell+2 \pi^{t} M^{-1} \eta_{j}\right) .
\end{aligned}
$$

If $j \neq 0$, then as above we have

$$
\left.D_{V}^{\beta}\left(\widehat{q}(\omega) \mathbf{P}\left({ }^{t} M^{-1} \omega\right)\right)\right|_{\omega=2 \pi \eta_{j}} G_{\Phi}\left(2 \pi^{t} M^{-1} \eta_{j}\right)=0
$$

and therefore $\left.D_{V}^{\beta}\left(\widehat{q}(\omega) \mathbf{P}\left({ }^{t} M^{-1} \omega\right)\right)\right|_{\omega=2 \pi \eta_{j}}=0$. If $j=0$, then

$$
\left.D_{V}^{\beta}\left(\widehat{q}(\omega) \mathbf{P}\left({ }^{t} M^{-1} \omega\right)\right)\right|_{\omega=0} \widehat{\Phi}(2 \pi \ell)+\lambda^{-\beta} \sum_{0 \leq \alpha<\beta}\left(\begin{array}{c}
\beta \\
\alpha
\end{array}\right) D_{V}^{\alpha} \widehat{q}(0) D_{V}^{\beta-\alpha} \widehat{\Phi}(2 \pi \ell)=0 .
$$

Since $\widehat{f}(\omega)=\widehat{q}(\omega) \widehat{\Phi}(\omega)$ and $D_{V}^{\beta} \widehat{f}(2 \pi \ell)=0, \ell \in \mathbb{Z}^{d}$,

$$
\sum_{0 \leq \alpha \leq \beta}\left(\begin{array}{c}
\beta \\
\alpha
\end{array}\right) D_{V}^{\alpha} \widehat{q}(0) D_{V}^{\beta-\alpha} \widehat{\Phi}(2 \pi \ell)=0 .
$$

Thus

$$
\left.D_{V}^{\beta}\left(\widehat{q}(\omega) \mathbf{P}\left({ }^{t} M^{-1} \omega\right)\right)\right|_{\omega=0} \widehat{\Phi}(2 \pi \ell)=\lambda^{-\beta} D_{V}^{\beta} \widehat{q}(0) \widehat{\Phi}(2 \pi \ell) .
$$

This leads to

$$
\left.D_{V}^{\beta}\left(\widehat{q}(\omega) \mathbf{P}\left({ }^{t} M^{-1} \omega\right)\right)\right|_{\omega=0} G_{\Phi}(0)=\lambda^{-\beta} D_{V}^{\beta} \widehat{q}(0) G_{\Phi}(0)
$$

and therefore

$$
\left.D_{V}^{\beta}\left(\widehat{q}(\omega) \mathbf{P}\left({ }^{t} M^{-1} \omega\right)\right)\right|_{\omega=0}=\lambda^{-\beta} D_{V}^{\beta} \widehat{q}(0) .
$$

It follows that (3.9) holds for $\beta$, so that the proof by induction is completed.

Denote by $\widetilde{\Phi}(x)$ the bi-infinite column from the integer shifts of $\Phi$ :

$$
\widetilde{\Phi}(x):={ }^{t}\left(\cdots,{ }^{t} \Phi(x+\ell), \cdots\right)_{\ell \in \mathbb{Z}^{d}},
$$

and by $L$ the bi-infinite matrix

$$
L:=\left(\mathbf{P}_{M \alpha-\beta}\right)_{\alpha, \beta \in \mathbb{Z}^{d}} .
$$

Then the refinement equation (1.1) can be written as

$$
L \widetilde{\Phi}(M x)=\widetilde{\Phi}(x) .
$$

The characterization of the accuracy order of $\Phi$ in terms of the eigenvalues and eigenvector structures of the infinite matrix $L$ were studied in [11], [25] and [17] for the case $d=1$. In [1], a similar characterization of the accuracy order of $\Phi$ was obtained based on the ergodic theorem for the multivariate case with arbitrary matrix dilations $M$ (no restriction on the diagonalization on $M$ ), and the coefficients $y_{\beta, i}(\kappa)$ for the polynomial reproducing $x^{\beta}=\sum_{i=1}^{r} \sum_{\kappa \in \mathbb{Z}^{d}} y_{\beta, i}(\kappa) \phi_{i}(x+\kappa)$ were determined explicitly. In the rest of this section, under the assumption that the 
integer shifts $\left(\phi_{i}(x-\ell), 1 \leq i \leq r, \ell \in \mathbb{Z}^{d}\right)$ of $\Phi$ are linearly independent, we will determine explicitly the coefficients $\mathbf{y}_{\ell}^{\beta}$ for the polynomial reproducing

$$
\sum_{\ell \in \mathbb{Z}^{d}} \mathbf{y}_{\ell}^{\beta} \Phi(x+\ell)=\left({ }^{t} V x\right)^{\beta}, \quad x \in \mathbb{R}^{d},|\beta|<k,
$$

where $V$ is the matrix defined by (3.1).

Theorem 3.7. Assume that $\Phi \in L^{2}\left(\mathbb{R}^{d}\right)$ is a compactly supported $(M, \mathbf{P})$ refinable vector and the integer shifts of $\Phi$ are linearly independent. If $\Phi$ has accuracy of order $k$ with $\mathbf{y}_{\ell}^{\beta}, \ell \in \mathbb{Z}^{d}, \beta \in \mathbb{Z}_{+}^{d},|\beta|<k$, being the $1 \times r$ complex vectors such that (3.11) holds, then $\mathbf{y}_{\ell}^{\beta}$ satisfy

(i) $\mathbf{y}_{\ell}^{\beta}=\sum_{0 \leq \alpha \leq \beta}\left(\begin{array}{c}\beta \\ \alpha\end{array}\right)\left(-{ }^{t} V \ell\right)^{\beta-\alpha} \mathbf{y}_{0}^{\alpha}$,

(ii) $\mathbf{y}^{\beta} L=\lambda^{-\beta} \mathbf{y}^{\beta}$, where $\mathbf{y}^{\beta}:=\left(\cdots, \mathbf{y}_{\ell}^{\beta}, \cdots\right)_{\ell \in \mathbb{Z}^{d}}$,

(iii) the vectors $\mathbf{y}_{0}^{\beta}, \beta \in \mathbb{Z}_{+}^{d},|\beta|<k$, satisfy the vanishing moment conditions (3.4).

Proof. Let $\mathbf{y}_{\ell}^{\beta}, \ell \in \mathbb{Z}^{d}, \beta \in \mathbb{Z}_{+}^{d},|\beta|<k$, be the complex vectors such that (3.11) holds. For any $\tau \in \mathbb{Z}^{d}$,

$$
\begin{aligned}
& \sum_{\ell \in \mathbb{Z}^{d}} \mathbf{y}_{\ell+\tau}^{\beta} \Phi(x+\ell)=\sum_{\ell \in \mathbb{Z}^{d}} \mathbf{y}_{\ell}^{\beta} \Phi(x-\tau+\ell)=\left({ }^{t} V(x-\tau)\right)^{\beta} \\
& =\sum_{0 \leq \alpha \leq \beta}\left(\begin{array}{c}
\beta \\
\alpha
\end{array}\right)\left(-{ }^{t} V \tau\right)^{\beta-\alpha}\left({ }^{t} V x\right)^{\alpha} \\
& =\sum_{0 \leq \alpha \leq \beta}\left(\begin{array}{c}
\beta \\
\alpha
\end{array}\right)\left(-{ }^{t} V \tau\right)^{\beta-\alpha} \sum_{\ell \in \mathbb{Z}^{d}} \mathbf{y}_{\ell}^{\alpha} \Phi(x+\ell) \\
& =\sum_{\ell \in \mathbb{Z}^{d}} \sum_{0 \leq \alpha \leq \beta}\left(\begin{array}{c}
\beta \\
\alpha
\end{array}\right)\left(-{ }^{t} V \tau\right)^{\beta-\alpha} \mathbf{y}_{\ell}^{\alpha} \Phi(x+\ell) .
\end{aligned}
$$

By the linear independence of the integer shifts of $\Phi$,

$$
\mathbf{y}_{\ell+\tau}^{\beta}=\sum_{0 \leq \alpha \leq \beta}\left(\begin{array}{c}
\beta \\
\alpha
\end{array}\right)\left(-{ }^{t} V \tau\right)^{\beta-\alpha} \mathbf{y}_{\ell}^{\alpha} .
$$

Let $\ell=0$; then (3.12) leads to (i).

For $\beta \in \mathbb{Z}_{+}^{d},|\beta|<k$, we have by (3.11)

$$
\left({ }^{t} V x\right)^{\beta}=\mathbf{y}^{\beta} \widetilde{\Phi}(x)=\mathbf{y}^{\beta} L \widetilde{\Phi}(M x)
$$

and

$$
\left({ }^{t} V x\right)^{\beta}=\lambda^{-\beta}\left(\Lambda^{t} V x\right)^{\beta}=\lambda^{-\beta}\left({ }^{t} V M x\right)^{\beta}=\lambda^{-\beta} \mathbf{y}^{\beta} \widetilde{\Phi}(M x) .
$$

By the linear independence of the integer shifts of $\Phi$ again,

$$
\mathbf{y}^{\beta} L=\lambda^{-\beta} \mathbf{y}^{\beta}, \quad \text { for } \beta \in \mathbb{Z}_{+}^{d},|\beta|<k .
$$

Finally, we verify (iii). Note that (3.13) can be written equivalently as

$$
\sum_{\ell \in \mathbb{Z}^{d}} \mathbf{y}_{\ell}^{\beta} \mathbf{P}_{M \ell-\ell^{\prime}}=\lambda^{-\beta} \mathbf{y}_{\ell^{\prime}}^{\beta}, \quad \text { for any } \ell^{\prime} \in \mathbb{Z}^{d}, \beta \in \mathbb{Z}_{+}^{d},|\beta|<k,
$$


and, in particular, for any $j, 0 \leq j \leq m-1$,

$$
\lambda^{-\beta} \mathbf{y}_{-\gamma_{j}}^{\beta}=\sum_{\ell \in \mathbb{Z}^{d}} \mathbf{y}_{\ell}^{\beta} \mathbf{P}_{M \ell+\gamma_{j}}=\sum_{\ell \in \mathbb{Z}^{d}} \sum_{0 \leq \alpha \leq \beta}\left(\begin{array}{c}
\beta \\
\alpha
\end{array}\right)\left(-{ }^{t} V \ell\right)^{\beta-\alpha} \mathbf{y}_{0}^{\alpha} \mathbf{P}_{M \ell+\gamma_{j}} .
$$

For any $\kappa \in \mathbb{Z}_{+}^{d},|\kappa|<k$, multiplying both side of (3.14) by

$$
\lambda^{\beta-\kappa}\left(-{ }^{t} V \gamma_{j}\right)^{\kappa-\beta}\left(\begin{array}{c}
\kappa \\
\beta
\end{array}\right)
$$

and summing over $\beta \leq \kappa$, one has by (3.12) and $\Lambda^{t} V={ }^{t} V M$,

$$
\begin{aligned}
\lambda^{-\kappa} \mathbf{y}_{0}^{\kappa}=\lambda^{-\kappa} \sum_{0 \leq \beta \leq \kappa}\left(\begin{array}{c}
\kappa \\
\beta
\end{array}\right)\left(-{ }^{t} V \gamma_{j}\right)^{\kappa-\beta} \mathbf{y}_{-\gamma_{j}}^{\beta} \\
=\sum_{\ell \in \mathbb{Z}^{d}} \sum_{0 \leq \beta \leq \kappa} \sum_{0 \leq \alpha \leq \beta}\left(\begin{array}{c}
\kappa \\
\beta
\end{array}\right)\left(\begin{array}{c}
\beta \\
\alpha
\end{array}\right) \lambda^{\beta-\kappa}\left(-{ }^{t} V \gamma_{j}\right)^{\kappa-\beta}\left(-{ }^{t} V \ell\right)^{\beta-\alpha} \mathbf{y}_{0}^{\alpha} \mathbf{P}_{M \ell+\gamma_{j}} \\
=\sum_{\ell \in \mathbb{Z}^{d}} \sum_{0 \leq \alpha \leq \kappa} \sum_{\alpha \leq \beta \leq \kappa}\left(\begin{array}{c}
\kappa \\
\alpha
\end{array}\right)\left(\begin{array}{c}
\kappa-\alpha \\
\beta-\alpha
\end{array}\right) \lambda^{\alpha-\kappa}\left(-{ }^{t} V \gamma_{j}\right)^{\kappa-\beta}\left(-{ }^{t} V M \ell\right)^{\beta-\alpha} \mathbf{y}_{0}^{\alpha} \mathbf{P}_{M \ell+\gamma_{j}} \\
=\sum_{\ell \in \mathbb{Z}^{d}} \sum_{0 \leq \alpha \leq \kappa}\left(\begin{array}{c}
\kappa \\
\alpha
\end{array}\right) \lambda^{\alpha-\kappa} \\
\cdot \sum_{0 \leq \tau \leq \kappa-\alpha}\left(\begin{array}{c}
\kappa-\alpha \\
\tau
\end{array}\right)\left(-{ }^{t} V \gamma_{j}\right)^{\kappa-\alpha-\tau}\left(-{ }^{t} V M \ell\right)^{\tau} \mathbf{y}_{0}^{\alpha} \mathbf{P}_{M \ell+\gamma_{j}} \\
=\sum_{\ell \in \mathbb{Z}^{d}} \sum_{0 \leq \alpha \leq \kappa}\left(\begin{array}{c}
\kappa \\
\alpha
\end{array}\right) \lambda^{\alpha-\kappa}\left(-{ }^{t} V\left(M \ell+\gamma_{j}\right)\right)^{\kappa-\alpha} \mathbf{y}_{0}^{\alpha} \mathbf{P}_{M \ell+\gamma_{j}} .
\end{aligned}
$$

Thus for any $\kappa \in \mathbb{Z}_{+}^{d},|\kappa|<k$,

$$
\sum_{0 \leq \alpha \leq \kappa}\left(\begin{array}{c}
\kappa \\
\alpha
\end{array}\right)(-\lambda)^{\alpha-\kappa} \mathbf{y}_{0}^{\alpha} \sum_{\ell \in \mathbb{Z}^{d}}\left({ }^{t} V\left(M \ell+\gamma_{j}\right)\right)^{\kappa-\alpha} \mathbf{P}_{M \ell+\gamma_{j}}=\lambda^{-\kappa} \mathbf{y}_{0}^{\kappa} .
$$

For any $s \in \mathbb{Z}_{+}, 0 \leq s \leq m-1$, multiplying both side of (3.15) by $e^{-2 \pi^{t} \eta_{s} M^{-1} \gamma_{j}}$ and summing over $j=0, \cdots, m-1$, one has by Lemma 2.1,

$$
\begin{aligned}
& \sum_{0 \leq \alpha \leq \kappa}\left(\begin{array}{c}
\kappa \\
\alpha
\end{array}\right)(-\lambda)^{\alpha-\kappa} \mathbf{y}_{0}^{\alpha} \sum_{j=0}^{m-1} \sum_{\ell \in \mathbb{Z}^{d}}\left({ }^{t} V\left(M \ell+\gamma_{j}\right)\right)^{\kappa-\alpha} \mathbf{P}_{M \ell+\gamma_{j}} e^{-2 \pi^{t} \eta_{s} M^{-1} \gamma_{j}} \\
& =\lambda^{-\kappa} \mathbf{y}_{0}^{\kappa} \sum_{j=0}^{m-1} e^{-2 \pi^{t} \eta_{s} M^{-1} \gamma_{j}}=m \lambda^{-\kappa} \mathbf{y}_{0}^{\kappa} \delta(s) .
\end{aligned}
$$

Thus

$$
\frac{1}{m} \sum_{0 \leq \alpha \leq \kappa}\left(\begin{array}{c}
\kappa \\
\alpha
\end{array}\right)(-\lambda)^{\alpha-\kappa} \mathbf{y}_{0}^{\alpha} \sum_{\ell^{\prime} \in \mathbb{Z}^{d}}\left({ }^{t} V \ell^{\prime}\right)^{\kappa-\alpha} \mathbf{P}_{\ell^{\prime}} e^{-2 \pi^{t} \eta_{s} M^{-1} \ell^{\prime}}=\lambda^{-\kappa} \mathbf{y}_{0}^{\kappa} \delta(s) .
$$


On the other hand, one has

$$
\begin{aligned}
& \sum_{0 \leq \alpha \leq \kappa}\left(\begin{array}{c}
\kappa \\
\alpha
\end{array}\right)(i \lambda)^{\alpha-\kappa} \mathbf{y}_{0}^{\alpha} D_{V}^{\kappa-\alpha} \mathbf{P}\left(2 \pi^{t} M^{-1} \eta_{s}\right) \\
= & \frac{1}{m} \sum_{0 \leq \alpha \leq \kappa}\left(\begin{array}{c}
\kappa \\
\alpha
\end{array}\right)(i \lambda)^{\alpha-\kappa} \mathbf{y}_{0}^{\alpha} \sum_{\ell \in \mathbb{Z}^{d}}\left(-i^{t} V \ell\right)^{\kappa-\alpha} \mathbf{P}_{\ell} e^{-i^{t} \eta_{s} M^{-1} \ell} \\
= & \frac{1}{m} \sum_{0 \leq \alpha \leq \kappa}\left(\begin{array}{c}
\kappa \\
\alpha
\end{array}\right)(-\lambda)^{\alpha-\kappa} \mathbf{y}_{0}^{\alpha} \sum_{\ell \in \mathbb{Z}^{d}}\left({ }^{t} V \ell\right)^{\kappa-\alpha} \mathbf{P}_{\ell} e^{-i^{t} \eta_{s} M^{-1} \ell} .
\end{aligned}
$$

Therefore for any $s \in \mathbb{Z}_{+}, 0 \leq s \leq m-1, \kappa \in \mathbb{Z}_{+}^{d},|\kappa|<k$,

$$
\sum_{0 \leq \alpha \leq \kappa}\left(\begin{array}{c}
\kappa \\
\alpha
\end{array}\right)(i \lambda)^{\alpha-\kappa} \mathbf{y}_{0}^{\alpha} D_{V}^{\kappa-\alpha} \mathbf{P}\left(2 \pi^{t} M^{-1} \eta_{s}\right)=\delta(s) \lambda^{-\kappa} \mathbf{y}_{0}^{\kappa}
$$

and the proof of (iii) is completed.

Remark 3.8. By Proposition 3.5, $\mathbf{y}_{0}^{\beta}, \beta \in \mathbb{Z}_{+}^{d},|\beta|<k$, are the unique vectors satisfying (3.4). Thus the unique coefficients $\mathbf{y}_{\ell}^{\beta}$ for the reproducing polynomial are given by (i) of Theorem 3.7, and they satisfy (ii) of Theorem 3.7.

\section{THE RESTRICTED TRANSITION OPERATOR}

Assume that $\mathbf{P}$ is a matrix refinement mask with $\operatorname{supp}\left\{\mathbf{P}_{\alpha}\right\} \subset[0, N]^{d}$ for some positive integer $N$, and $\Phi$ is a compactly supported $(M, \mathbf{P})$ refinable vector. It was shown in Section 2 that to decide whether $\Phi$ is stable (orthogonal) or not, we need only to check the properties of the spectra (eigenvalues) and the 1-eigenvector of the restriction $\left.\mathbf{T}\right|_{\mathbb{H}}$ of the transition operator $\mathbf{T}$ to $\mathbb{H}$, where $\mathbb{H}$ is the finite dimensional space defined by (1.5) and $\mathbf{T}$ is the transition operator defined by (1.3). It is useful in practice to transfer equivalently the restricted operator $\left.\mathbf{T}\right|_{\mathbb{H}}$ to a finite matrix, since eigenvalues and eigenvectors of a finite matrix can be computed directly. In this section, we give the representing matrix $\mathcal{T}$ of $\left.\mathbf{T}\right|_{\mathbb{H}}$, and then study the spectral properties of $\mathbf{T}$.

For $H(\omega)=\sum_{\ell \in[\Omega]} H_{\ell} e^{-i \ell \omega} \in \mathbb{H}$, by $(2.5)$, under the basis $\left\{e^{-i \ell \omega}\right\}_{\ell \in[\Omega]}$ of $\mathbb{H}, \mathbf{T}$ transfers the sequence $\left\{H_{\ell}\right\}_{\ell \in[\Omega]}$ into another sequence:

$$
\left\{m^{-1} \sum_{\ell \in[\Omega]} \sum_{\kappa \in[0, N]^{d}} \mathbf{P}_{\kappa} H_{\ell}^{t} \mathbf{P}_{\kappa-(M \tau-\ell)}\right\}_{\tau \in[\Omega]} .
$$

Now let us look at the matrices of the form $\mathbf{P}_{\kappa} H_{\ell}{ }^{t} \mathbf{P}_{\tau}$. Let $Q=(Q(1), \cdots, Q(r))$ be an $r \times r$ matrix with $Q(j)$ the $j$ th column, and define an $r^{2} \times 1 \operatorname{vector} \operatorname{vec}(Q)$ by

$$
\operatorname{vec}(Q):={ }^{t}\left({ }^{t} Q(1), \cdots,{ }^{t} Q(r)\right)
$$

Then we have the following lemma.

Lemma 4.1. Let $P, Q, H$ be $r \times r$ matrices, then

$$
\operatorname{vec}\left(P H^{t} Q\right)=(Q \otimes P) \operatorname{vec}(H),
$$

where $Q \otimes P=\left(q_{i j} P\right)_{1 \leq i, j \leq r}$, the Kronecker product of matrices $Q$ and $P$. 
Proof. Let $P(i), H(i)$ denote the $i$ th column of $P$ and $H$, respectively, and let $q_{i j}$ be the $(i, j)$-entry of $Q$. Then the $j$ th column of $P H^{t} Q$ is

$$
P H\left(q_{j i}\right)_{i=1}^{r}=\sum_{i=1}^{r} q_{j i} P H(i)=\left(q_{j 1} P, \cdots, q_{j r} P\right)^{t}\left({ }^{t} H(1), \cdots,{ }^{t} H(r)\right) .
$$

Thus

$$
\begin{aligned}
& \operatorname{vec}\left(P H^{t} Q\right)={ }^{t}\left({ }^{t}\left(P H\left(q_{1 i}\right)_{i=1}^{r}\right), \cdots,{ }^{t}\left(P H\left(q_{r i}\right)_{i=1}^{r}\right)\right) \\
& \quad=\left(q_{j i} P\right)_{1 \leq j \leq r, 1 \leq i \leq r}{ }^{t}\left({ }^{t} H(1), \cdots,{ }^{t} H(r)\right)=(Q \otimes P) \operatorname{vec}(H) .
\end{aligned}
$$

About formula (4.1) for more general matrices, one can refer to [12], and in particular, one has that, for any $1 \times r$ vectors $\mathbf{v}, \mathbf{u}$ and $r \times r$ matrix $Q$,

$$
(\mathbf{v} \otimes \mathbf{u}) \operatorname{vec}(Q)=\mathbf{u} Q^{t} \mathbf{v},
$$

where $\mathbf{v} \otimes \mathbf{u}$ denotes the Kronecker product of $\mathbf{v}, \mathbf{u}$.

For $j \in \mathbb{Z}^{d}$, define $r^{2} \times r^{2}$ matrices

$$
\mathcal{A}_{j}:=m^{-1} \sum_{\ell \in[0, N]^{d}} \mathbf{P}_{\ell-j} \otimes \mathbf{P}_{\ell}
$$

and define an $\left(r^{2}|[\Omega]|\right) \times\left(r^{2}|[\Omega]|\right)$ matrix

$$
\mathcal{T}:=\left(\mathcal{A}_{M i-j}\right)_{i, j \in[\Omega]} .
$$

For $f=\sum_{j \in[\Omega]} f_{j} e^{-i \omega j} \in \mathbb{H}$, let $\operatorname{vec}(f)$ be the $\left(r^{2}|[\Omega]|\right) \times 1$ vector defined by

$$
\operatorname{vec}(f):={ }^{t}\left(\cdots,{ }^{t}\left(\operatorname{vec}\left(f_{j}\right)\right), \cdots\right)_{j \in[\Omega]} .
$$

Then from (2.5) and (4.1), for any $\tau \in[\Omega]$,

$$
\begin{aligned}
& \operatorname{vec}\left((\mathbf{T} H)_{\tau}\right)=m^{-1} \sum_{\ell \in[\Omega]} \sum_{\kappa \in[0, N]^{d}} \operatorname{vec}\left(\mathbf{P}_{\kappa} H_{\ell}{ }^{t} \mathbf{P}_{\kappa-(M \tau-\ell)}\right) \\
& =m^{-1} \sum_{\ell \in[\Omega]} \sum_{\kappa \in[0, N]^{d}}\left(\mathbf{P}_{\kappa-(M \tau-\ell)} \otimes \mathbf{P}_{\kappa}\right) \operatorname{vec}\left(H_{\ell}\right) \\
& =\sum_{\ell \in[\Omega]} \mathcal{A}_{M \tau-\ell} \operatorname{vec}\left(H_{\ell}\right)=\left(\mathcal{T}_{\operatorname{vec}}(H)\right)(\tau) .
\end{aligned}
$$

Hence we have

Theorem 4.2. The restriction of the transition operator $\mathbf{T}$ to $\mathbb{H}$ is equivalent to the matrix $\mathcal{T}$ defined by (4.3) under the basis $\left\{e^{-i \omega \ell}\right\}_{\ell \in[\Omega]}$ of $\mathbb{H}$, and for $H \in \mathbb{H}$

$$
\operatorname{vec}(\mathbf{T} H)=\mathcal{T} \operatorname{vec}(H) .
$$

Lemma 2.2, Theorem 2.11, Theorem 2.12 and Theorem 4.2 lead to the following two corollaries.

Corollary 4.3. The refinement equation (1.1) has a compactly supported solution which is stable if and only if the following conditions hold:

(i) the matrix $\mathbf{P}(0)$ satisfies Condition $E$,

(ii) for the left (row) 1-eigenvector $\mathbf{l}$ of $\mathbf{P}(0), \mathbf{l} \mathbf{P}\left(2 \pi^{t} M^{-1} \eta_{j}\right)=0,1 \leq j \leq m-1$,

(iii) the finite matrix $\mathcal{T}$ satisfies Condition $E$ and the corresponding right 1eigenvector $\mathbf{v}$ is such that $H_{0}(\omega)$ is positive (or negative) definite on $\mathbb{T}^{d}$, where $H_{0}(\omega)$ is the unique matrix function in $\mathbb{H}$ satisfying vec $\left(H_{0}\right)=\mathbf{v}$. 
Corollary 4.4. The refinement equation (1.1) has a compactly supported solution which is orthogonal if and only if the following conditions hold:

(i) the mask $\mathbf{P}$ is a $C Q F$,

(ii) the matrix $\mathbf{P}(0)$ satisfies Condition $E$,

(iii) for the left (row) 1-eigenvector $\mathbf{l}$ of $\mathbf{P}(0), \mathbf{P}\left(2 \pi^{t} M^{-1} \eta_{j}\right)=0,1 \leq j \leq m-1$,

(iv) the finite matrix $\mathcal{T}$ satisfies Condition $E$.

By (4.4), $\mathbf{v}$ is an eigenvector of $\mathcal{T}$ if and only if the matrix-valued function $H(\omega)$ in $\mathbb{H}$ with $\operatorname{vec}(H)=\mathbf{v}$ is an eigenvector of $\mathbf{T}$, and furthermore $\mathbf{v}, H(\omega)$ correspond to the same eigenvalue. Therefore to study the spectral properties of $\mathbf{T}$, we need only to consider those of the matrix $\mathcal{T}$. In the rest of this section, we will discuss the spectral properties of $\mathcal{T}$. In the following, we will assume that the eigenvalues of the dilation matrix $M$ are nondegenerate, and let $\lambda_{j}, 1 \leq j \leq d$, be the eigenvalues of $M$. Let $V$ denote the matrix defined by (3.1). We also assume that $\mathbf{P}$ satisfies the vanishing moment condition of order $k$ for some positive integer $k$, i.e. $\mathbf{P}$ satisfies (3.4) for some vectors $\mathbf{l}_{0}^{\beta}, \beta \in \mathbb{Z}_{+}^{d},|\beta|<k$, with $\mathbf{l}_{0}^{0} \neq 0$.

Let $k_{0} \in \mathbb{Z}_{+}, k_{0} \leq k$, be the largest integer such that there exist $1 \times r$ complex vectors $\mathbf{l}_{0}^{\beta}, \beta \in \mathbb{Z}_{+}^{d}, k \leq|\beta| \leq k+k_{0}-1$, satisfying

$$
\sum_{0 \leq \alpha \leq \beta}\left(\begin{array}{c}
\beta \\
\alpha
\end{array}\right)(i \lambda)^{\alpha-\beta} \mathbf{l}_{0}^{\alpha} D_{V}^{\beta-\alpha} \mathbf{P}(0)=\lambda^{-\beta} \mathbf{l}_{0}^{\beta} .
$$

If all the numbers $\lambda^{-\beta}, k \leq|\beta| \leq k+k_{0}-1$, are not eigenvalues of $\mathbf{P}(0)$ for some $k_{0} \in \mathbb{Z}_{+}$, then the vectors $\mathbf{l}_{0}^{\beta}, \beta \in \mathbb{Z}_{+}^{d}, k \leq|\beta| \leq k+k_{0}-1$, can be chosen iteratively by

$$
\mathbf{l}_{0}^{\beta}\left(\lambda^{-\beta} \mathbf{I}_{r}-\mathbf{P}(0)\right)=\sum_{0 \leq \alpha<\beta}\left(\begin{array}{c}
\beta \\
\alpha
\end{array}\right)(i \lambda)^{\alpha-\beta} \mathbf{l}_{0}^{\alpha}\left(D_{V}^{\beta-\alpha} \mathbf{P}\right)(0) .
$$

For the case $r=1$, since $\mathbf{P}(0)=1, k_{0}=k$.

Let $B(\omega)=\sum_{\ell \in \mathbb{Z}_{+}^{d},|\ell|<k+k_{0}} B_{\ell} e^{i \ell \omega}$ be the vector trigonometric polynomial satisfying

$$
D_{V}^{\beta} B(0)=i^{|\beta|} \mathbf{l}_{0}^{\beta}, \quad \beta \in \mathbb{Z}_{+}^{d},|\beta|<k+k_{0} .
$$

The coefficients $B_{\kappa}, 1 \times r$ vectors, can be gotten by the following equations:

$$
\sum_{|\ell|<k+k_{0}}\left({ }^{t} V \ell\right)^{\beta} B_{\ell}=\mathbf{l}_{0}^{\beta}, \quad \beta \in \mathbb{Z}_{+}^{d},|\beta|<k+k_{0} .
$$

By (3.2), for any $j \in \mathbb{Z}_{+}, 0 \leq j \leq m-1$,

$$
\begin{aligned}
& \left.D_{V}^{\beta}\left(B\left({ }^{t} M \omega\right) \mathbf{P}(\omega)\right)\right|_{\omega=2 \pi^{t} M^{-1} \eta_{j}} \\
& =\left.\sum_{0 \leq \alpha \leq \beta}\left(\begin{array}{c}
\beta \\
\alpha
\end{array}\right) \lambda^{\alpha}\left(\left(D_{V}^{\alpha} B\right)\left({ }^{t} M \omega\right) D_{V}^{\beta-\alpha} \mathbf{P}(\omega)\right)\right|_{\omega=2 \pi^{t} M^{-1} \eta_{j}} \\
& =\left.\sum_{0 \leq \alpha \leq \beta}\left(\begin{array}{c}
\beta \\
\alpha
\end{array}\right) \lambda^{\alpha}\left(D_{V}^{\alpha} B\right)(0) D_{V}^{\beta-\alpha} \mathbf{P}(\omega)\right|_{\omega=2 \pi^{t} M^{-1} \eta_{j}} \\
& =\sum_{0 \leq \alpha \leq \beta}\left(\begin{array}{c}
\beta \\
\alpha
\end{array}\right)(i \lambda)^{\alpha} \mathbf{l}_{0}^{\alpha} D_{V}^{\beta-\alpha} \mathbf{P}\left(2 \pi^{t} M^{-1} \eta_{j}\right) .
\end{aligned}
$$


Thus the vanishing moment conditions (3.4) and (4.5) can be written equivalently in the forms

$$
\begin{array}{r}
\left.D_{V}^{\beta}\left(B\left({ }^{t} M \omega\right) \mathbf{P}(\omega)\right)\right|_{\omega=2 \pi^{t} M^{-1} \eta_{j}}=\delta(j) D_{V}^{\beta} B(0), \\
\beta \in \mathbb{Z}_{+}^{d},|\beta|<k, 0 \leq j<m,
\end{array}
$$

and

$$
\left.D_{V}^{\beta}\left(B\left({ }^{t} M \omega\right) \mathbf{P}(\omega)\right)\right|_{\omega=0}=D_{V}^{\beta} B(0), \quad \beta \in \mathbb{Z}_{+}^{d}, k \leq|\beta|<k+k_{0} .
$$

Let $\mathbf{l}_{0}^{\beta}, \beta \in \mathbb{Z}_{+}^{d},|\beta|<k+k_{0}$, be the row vectors satisfying (3.4) and (4.5). For $\kappa \in \mathbb{Z}^{d}$, define row vectors $\mathbf{l}_{\kappa}^{\beta}$ by

$$
\mathbf{l}_{\kappa}^{\beta}:=\sum_{0 \leq \alpha \leq \beta}\left(\begin{array}{c}
\beta \\
\alpha
\end{array}\right)\left(-{ }^{t} V \kappa\right)^{\beta-\alpha} \mathbf{l}_{0}^{\alpha}, \quad \text { for } \beta \in \mathbb{Z}_{+}^{d},|\beta|<k+k_{0},
$$

and then define $1 \times\left(r^{2}|[\Omega]|\right)$ vectors $\mathbf{L}_{\Omega}^{\beta}$ by

$$
\mathbf{L}_{\Omega}^{\beta}:=\left(\cdots, \mathbf{l}^{\beta}(\kappa), \cdots\right)_{\kappa \in[\Omega]}
$$

with

$$
\mathbf{l}^{\beta}(\kappa):=\sum_{0 \leq \alpha \leq \beta}(-1)^{\alpha}\left(\begin{array}{c}
\beta \\
\alpha
\end{array}\right) \overline{\mathbf{l}}_{-\kappa}^{\alpha} \otimes \mathbf{l}_{0}^{\beta-\alpha}, \quad \kappa \in \mathbb{Z}^{d} .
$$

Lemma 4.5. For any $\beta \in \mathbb{Z}_{+}^{d},|\beta|<k+k_{0}$, let $\mathbf{L}_{\Omega}^{\beta}$ be the vectors defined by (4.10). Then for any $H \in \mathbb{H}$

$$
\mathbf{L}_{\Omega}^{\beta} \operatorname{vec}(H)=\left.(-i)^{|\beta|} D_{V}^{\beta}\left(B(\omega) H(\omega) B^{*}(\omega)\right)\right|_{\omega=0} .
$$

Proof. By (4.2), for any $\beta \in \mathbb{Z}_{+}^{d},|\beta|<k+k_{0}$, and any $H \in \mathbb{H}$

$$
\begin{aligned}
& \mathbf{L}_{\Omega}^{\beta} \operatorname{vec}(H)=\sum_{\kappa} \mathbf{l}^{\beta}(\kappa) \operatorname{vec}\left(H_{\kappa}\right)=\sum_{\kappa} \sum_{0 \leq \alpha \leq \beta}(-1)^{\alpha}\left(\begin{array}{c}
\beta \\
\alpha
\end{array}\right) \mathbf{l}_{0}^{\beta-\alpha} H(\kappa)\left(\mathbf{l}_{-\kappa}^{\alpha}\right)^{*} \\
& =\sum_{\kappa} \sum_{0 \leq \alpha \leq \beta}(-1)^{\alpha}\left(\begin{array}{c}
\beta \\
\alpha
\end{array}\right) \mathbf{l}_{0}^{\beta-\alpha} H(\kappa) \sum_{0 \leq \gamma \leq \alpha}\left({ }^{t} V \kappa\right)^{\gamma}\left(\begin{array}{c}
\alpha \\
\gamma
\end{array}\right)\left(\mathbf{l}_{0}^{\alpha-\gamma}\right)^{*} \\
& =\sum_{\kappa} \sum_{0 \leq \alpha \leq \beta} \sum_{0 \leq \gamma \leq \alpha}(-1)^{\alpha}\left(\begin{array}{c}
\beta \\
\alpha
\end{array}\right) \\
& \quad \cdot\left({ }^{t} V \kappa\right)^{\gamma}\left(\begin{array}{c}
\alpha \\
\gamma
\end{array}\right)(-i)^{|\beta-\alpha|} D_{V}^{\beta-\alpha} B(0) H(\kappa) i^{|\alpha-\gamma|} D_{V}^{\alpha-\gamma} B^{*}(0) \\
& =(-i)^{|\beta|} \sum_{0 \leq \alpha \leq \beta} \sum_{0 \leq \gamma \leq \alpha}\left(\begin{array}{c}
\beta \\
\alpha
\end{array}\right)\left(\begin{array}{c}
\alpha \\
\gamma
\end{array}\right) D_{V}^{\beta-\alpha} B(0) \sum_{\kappa}\left(-i^{t} V \kappa\right)^{\gamma} H(\kappa) D_{V}^{\alpha-\gamma} B^{*}(0) \\
& =(-i)^{|\beta|} \sum_{0 \leq \alpha \leq \beta} \sum_{0 \leq \gamma \leq \alpha}\left(\begin{array}{c}
\beta \\
\alpha
\end{array}\right)\left(\begin{array}{c}
\alpha \\
\gamma
\end{array}\right) D_{V}^{\beta-\alpha} B(0) D_{V}^{\gamma} H(0) D_{V}^{\alpha-\gamma} B^{*}(0) \\
& =\left.(-i)^{|\beta|} D_{V}^{\beta}\left(B(\omega) H(\omega) B^{*}(\omega)\right)\right|_{\omega=0} .
\end{aligned}
$$

For $\beta \in \mathbb{Z}_{+}^{d},|\beta|<k+k_{0}$, denote

$$
E_{\beta}:=\left\{\beta^{\prime}: \lambda^{\beta^{\prime}}=\lambda^{\beta}, \beta^{\prime} \in \mathbb{Z}_{+}^{d},\left|\beta^{\prime}\right|<k+k_{0}\right\} .
$$


Theorem 4.6. For any $\beta \in \mathbb{Z}_{+}^{d},|\beta|<k+k_{0}$, let $\mathbf{L}_{\Omega}^{\beta}$ be the vectors defined by (4.10). Then

$$
\mathbf{L}_{\Omega}^{\beta} \mathcal{T}=\lambda^{-\beta} \mathbf{L}_{\Omega}^{\beta}
$$

If there exists a $\beta^{\prime} \in E_{\beta}$ such that $\mathbf{L}_{\Omega}^{\beta^{\prime}} \neq \mathbf{0}$, then $\lambda^{-\beta}$ is an eigenvalue of $\mathcal{T}$ with a corresponding left eigenvector $\mathbf{L}_{\Omega}^{\beta^{\prime}}$.

Proof. We need only to show that for any $H \in \mathbb{H}$,

$$
\mathbf{L}_{\Omega}^{\beta} \mathcal{T} \operatorname{vec}(H)=\lambda^{-\beta} \mathbf{L}_{\Omega}^{\beta} \operatorname{vec}(H) .
$$

In fact, by (4.4) and Lemma 4.5,

$$
\begin{aligned}
(i \lambda){ }^{\beta} \mathbf{L}_{\Omega}^{\beta} \mathcal{T} \operatorname{vec}(H)=(i \lambda){ }^{\beta} \mathbf{L}_{\Omega}^{\beta} \operatorname{vec}(\mathbf{T} H) & \left.D_{V}^{\beta}\left(B\left({ }^{t} M \omega\right)(\mathbf{T} H)\left({ }^{t} M \omega\right) B^{*}\left({ }^{t} M \omega\right)\right)\right|_{\omega=0} \\
= & \sum_{j=0}^{m-1} D_{V}^{\beta}\left(B\left({ }^{t} M \omega\right) \mathbf{P}\left(2 \pi \omega+2 \pi^{t} M^{-1} \eta_{j}\right)\right. \\
& \left.\cdot H\left(2 \pi \omega+2 \pi^{t} M^{-1} \eta_{j}\right) \mathbf{P}\left(2 \pi \omega+2 \pi^{t} M^{-1} \eta_{j}\right)^{*} B^{*}\left({ }^{t} M \omega\right)\right)\left.\right|_{\omega=0} \\
= & \left.\sum_{j=0}^{m-1} \sum_{0 \leq \alpha \leq \beta} \sum_{0 \leq \gamma \leq \alpha}\left(\begin{array}{c}
\beta \\
\alpha
\end{array}\right)\left(\begin{array}{c}
\alpha \\
\gamma
\end{array}\right) D_{V}^{\alpha}\left(B\left({ }^{t} M \omega\right) \mathbf{P}(\omega)\right)\right|_{\omega=2 \pi^{t} M^{-1} \eta_{j}} \\
& \left.\left.\cdot D_{V}^{\gamma} H(\omega)\right|_{\omega=2 \pi^{t} M^{-1} \eta_{j}} D_{V}^{\beta-\alpha-\gamma}\left(B\left({ }^{t} M \omega\right) \mathbf{P}(\omega)\right)^{*}\right|_{\omega=2 \pi^{t} M^{-1} \eta_{j}} .
\end{aligned}
$$

Since for any $\beta, \alpha, \gamma \in \mathbb{Z}_{+}^{d}$ with $|\beta|<k+k_{0}$ and $\gamma \leq \alpha \leq \beta$, we have the inequality $\min (|\alpha|,|\beta-\alpha-\gamma|)<k$, it follows, from (4.7) and (4.8), that

$$
\begin{aligned}
(i \lambda)^{\beta} \mathbf{L}_{\Omega}^{\beta} \mathcal{T} \operatorname{vec}(H)= & \left.\sum_{0 \leq \alpha \leq \beta} \sum_{0 \leq \gamma \leq \alpha}\left(\begin{array}{c}
\beta \\
\alpha
\end{array}\right)\left(\begin{array}{c}
\alpha \\
\gamma
\end{array}\right) D_{V}^{\alpha}\left(B\left({ }^{t} M \omega\right) \mathbf{P}(\omega)\right)\right|_{\omega=0} \\
& \left.\left.\cdot D_{V}^{\gamma} H(\omega)\right|_{\omega=0} D_{V}^{\beta-\alpha-\gamma}\left(B\left({ }^{t} M \omega\right) \mathbf{P}(\omega)\right)^{*}\right|_{\omega=0} \\
= & \sum_{0 \leq \alpha \leq \beta} \sum_{0 \leq \gamma \leq \alpha}\left(\begin{array}{c}
\beta \\
\alpha
\end{array}\right)\left(\begin{array}{c}
\alpha \\
\gamma
\end{array}\right) D_{V}^{\alpha} B(0) D_{V}^{\gamma} H(0) D_{V}^{\beta-\alpha-\gamma} B^{*}(0) \\
= & \left.D_{V}^{\beta}\left(B(\omega) H(\omega) B^{*}(\omega)\right)\right|_{\omega=0}=i^{|\beta|} \mathbf{L}_{\Omega}^{\beta} \operatorname{vec}(H) .
\end{aligned}
$$

Therefore $\mathbf{L}_{\Omega}^{\beta} \mathcal{T} \operatorname{vec}(H)=\lambda^{-\beta} \mathbf{L}_{\Omega}^{\beta} \operatorname{vec}(H)$. The second statement of Theorem 4.2 follows from (4.11), and the proof of Theorem 4.6 is completed.

Since $\mathbf{L}_{\Omega}^{0}=\left(\mathbf{l}_{0}^{0}, \cdots, \mathbf{l}_{0}^{0}\right) \neq 0,1$ is an eigenvalue of $\mathbf{T}$. In the case $r=1, d=$ $1, M=(2)$, then $\Omega=[-N, N]$ and $k_{0}=k$. For any $n \in \mathbb{Z}_{+}, n \leq 2 k-1$, the vector $\left((-N)^{n}, \cdots,(-1)^{n}, 0^{n}, 1^{n}, \cdots, N^{n}\right)$ (with $\left.0^{n}:=\delta(n)\right)$ is the generalized left eigenvector of the eigenvalue $2^{-n}$ of $\mathcal{T}$, and hence $2^{-n}, 0 \leq n \leq 2 k-1$, are eigenvalues of $\mathbf{T}$ (see [5]). Theorem 4.6 says that for $\beta \in \mathbb{Z}_{+}^{d},|\beta|<k+k_{0}$, if there exits $\beta^{\prime} \in E_{\beta}$ such that $\mathbf{L}_{\Omega}^{\beta^{\prime}} \neq 0$, then $\lambda^{-\beta}$ is an eigenvalue of $\mathbf{T}$. If the refinement equation (1.1) has a compactly supported solution $\Phi$ with $\Phi \in W^{s}\left(\mathbb{R}^{d}\right)$ for some $s \geq 0$, then one can show similarly as in [19] that $\mathbf{L}_{\Omega}^{\beta} \neq 0$ for $\beta \in$ $\mathbb{Z}_{+}^{d},|\beta| \leq \min \left(k+k_{0}-1,2 s\right)$, and hence $\lambda^{-\beta}$ are eigenvalues of $\mathbf{T}$. In this paper, for $s \geq 0$, we say a vector-valued function $f={ }^{t}\left(f_{1}, \cdots, f_{r}\right)$ is in the Sobolev space $W^{s}\left(\mathbb{R}^{d}\right)$ if every component $f_{j}$ of $f$ satisfies $\left(1+|\omega|^{2}\right)^{\frac{s}{2}} \widehat{f}_{j}(\omega) \in L^{2}\left(\mathbb{R}^{d}\right), 1 \leq j \leq r$. 
The vectors $\mathbf{L}_{\Omega}^{\beta}$ play an important role in estimating the Sobolev regularity of the refinable vector $\Phi$, which will be done in the next section.

\section{Sobolev Regularity estimates}

Assume that $\mathbf{P}\left(\left\{\mathbf{P}_{\alpha}\right\}\right)$ is a matrix refinement mask satisfying (3.4) and (4.5) for some positive integers $k, k_{0}$ with $k_{0} \leq k$, and $\Phi$ is a compactly supported $(M, \mathbf{P})$ refinable vector. Suppose $\operatorname{supp}\left\{\mathbf{P}_{\alpha}\right\} \subset[0, N]^{d}$, and let $\mathbb{H}$ be the space defined by (1.5). In this section, we will estimate the Sobolev regularity of $\Phi$ in terms of the spectral radius of the restriction of the transition operator $\mathbf{T}$ to an invariant subspace $\mathbb{H}^{0}$ of $\mathbb{H}$.

For $j \in \mathbb{Z}_{+}, 1 \leq j \leq r$, and $\alpha \in \mathbb{Z}_{+}^{d},|\alpha|<k$, let ${ }_{j} \mathbf{l}_{\Omega}^{\alpha},{ }_{j} \mathbf{r}_{\Omega}^{\alpha}$ be the $1 \times\left(r^{2}|[\Omega]|\right)$ vectors defined by

$$
{ }_{j} \mathbf{l}_{\Omega}^{\alpha}:=\left(\cdots,{ }_{j} \mathbf{l}^{\alpha}(\kappa), \cdots\right)_{\kappa \in[\Omega]}, \quad{ }_{j} \mathbf{r}_{\Omega}^{\alpha}:=\left(\cdots,{ }_{j} \mathbf{r}^{\alpha}(\kappa), \cdots\right)_{\kappa \in[\Omega]}
$$

with

$$
{ }_{j} \mathbf{l}^{\alpha}(\kappa):={ }^{t} \mathbf{e}_{j} \otimes \mathbf{l}_{\kappa}^{\alpha}, \quad{ }_{j} \mathbf{r}^{\alpha}(\kappa):=\overline{\mathbf{l}}_{-\kappa}^{\alpha} \otimes{ }^{t} \mathbf{e}_{j}, \quad \kappa \in \mathbb{Z}^{d} .
$$

Lemma 5.1. For $j, 1 \leq j \leq r$, and $\alpha \in \mathbb{Z}_{+}^{d},|\alpha| \leq k-1$, let ${ }_{j} \mathbf{l}_{\Omega}^{\alpha},{ }_{j} \mathbf{r}_{\Omega}^{\alpha}$ be the row vectors defined by (5.1). Then for any $H \in \mathbb{H}$,

$$
\begin{aligned}
& { }_{j} \mathbf{1}_{\Omega}^{\alpha} \operatorname{vec}(H)=\left.i^{\alpha} D_{V}^{\alpha}\left(B(\omega) H(\omega) \mathbf{e}_{j}\right)\right|_{\omega=0}, \\
& { }_{j} \mathbf{r}_{\Omega}^{\alpha} \operatorname{vec}(H)=\left.(-i)^{\alpha} D_{V}^{\alpha}\left({ }^{t} \mathbf{e}_{j} H(\omega) B^{*}(\omega)\right)\right|_{\omega=0} .
\end{aligned}
$$

Proof. For any $H \in \mathbb{H}$ with $H(\omega)=\sum_{\kappa \in[\Omega]} H_{\kappa} e^{-i \kappa \omega}$,

$$
\begin{aligned}
& \left.D_{V}^{\alpha}\left(B(\omega) H(\omega) \mathbf{e}_{j}\right)\right|_{\omega=0}=\sum_{0 \leq \gamma \leq \alpha}\left(\begin{array}{c}
\alpha \\
\gamma
\end{array}\right) D_{V}^{\gamma} B(0) D_{V}^{\alpha-\gamma} H(0) \mathbf{e}_{j} \\
& =i^{\alpha} \sum_{\kappa} \sum_{0 \leq \gamma \leq \alpha}\left(\begin{array}{c}
\alpha \\
\gamma
\end{array}\right)\left(-{ }^{t} V \kappa\right)^{\alpha-\gamma} \mathbf{l}_{0}^{\gamma} H_{\kappa} \mathbf{e}_{j}=i^{\alpha} \sum_{\kappa} \mathbf{l}_{\kappa}^{\alpha} H_{\kappa} \mathbf{e}_{j} \\
& =i^{\alpha} \sum_{\kappa}\left({ }^{t} \mathbf{e}_{j} \otimes \mathbf{l}_{\kappa}^{\alpha}\right) \operatorname{vec}\left(H_{\kappa}\right)=i^{\alpha}{ }_{j} \mathbf{l}_{\Omega}^{\alpha} \operatorname{vec}(H) .
\end{aligned}
$$

The proof of the second formula is similar, and it is omitted here.

Let $\mathbb{H}^{0}$ be the subspace of $\mathbb{H}$ defined by

$$
\begin{aligned}
\mathbb{H}^{0}:= & \left\{H \in \mathbb{H}: \mathbf{L}_{\Omega}^{\beta} \operatorname{vec}(H)=0,{ }_{j} \mathbf{l}_{\Omega}^{\alpha} \operatorname{vec}(H)=0\right. \text { and } \\
& \left.{ }_{j} \mathbf{r}_{\Omega}^{\alpha} \operatorname{vec}(H)=0, \forall \beta, \alpha \in \mathbb{Z}_{+}^{d},|\beta|<k+k_{0},|\alpha|<k, 1 \leq j \leq r\right\} .
\end{aligned}
$$

Proposition 5.2. The subspace $\mathbb{H}^{0}$ of $\mathbb{H}$ defined by (5.2) is invariant under $\mathbf{T}$.

Proof. By Theorem 4.6, for any $H \in \mathbb{H}^{0}$ and $\beta \in \mathbb{Z}_{+}^{d},|\beta|<k+k_{0}$,

$$
\mathbf{L}_{\Omega}^{\beta} \operatorname{vec}(\mathbf{T} H)=\mathbf{L}_{\Omega}^{\beta} \mathcal{T} \operatorname{vec}(H)=\lambda^{-\beta} \mathbf{L}_{\Omega}^{\beta} \operatorname{vec}(H)=0 .
$$

By Lemma 5.1, for any $\alpha \in \mathbb{Z}_{+}^{d},|\alpha|<k$, the equalities ${ }_{j} \mathbf{l}_{\Omega}^{\alpha} \operatorname{vec}(H)=0$ and ${ }_{j} \mathbf{r}_{\Omega}^{\alpha} \operatorname{vec}(H)=0$ for all $j, 1 \leq j \leq r$, are equivalent to $\left.D_{V}^{\alpha}(B(\omega) H(\omega))\right|_{\omega=0}=0$ and $\left.D_{V}^{\alpha}\left(H(\omega) B^{*}(\omega)\right)\right|_{\omega=0}=0$, respectively. One can check by (4.7) and (4.8) that $\left.D_{V}^{\alpha}(B(\omega) \mathbf{T} H(\omega))\right|_{\omega=0}=0\left(\left.D_{V}^{\alpha}\left(\mathbf{T} H(\omega) B^{*}(\omega)\right)\right|_{\omega=0}=0\right.$ resp. $)$ for all $\alpha \in$ $\mathbb{Z}_{+}^{d},|\alpha|<k$, if $\left.D_{V}^{\alpha}(B(\omega) H(\omega))\right|_{\omega=0}=0\left(\left.D_{V}^{\alpha}\left(H(\omega) B^{*}(\omega)\right)\right|_{\omega=0}=0\right.$ resp. $)$ for $\alpha \in \mathbb{Z}_{+}^{d},|\alpha|<k$. Thus $\mathbb{H}^{0}$ is invariant under $\mathbf{T}$. 
Let $\left.\mathbf{T}\right|_{\mathbb{H} 0}$ denote the restriction of $\mathbf{T}$ to $\mathbb{H}^{0}$. We will want to find the Sobolev regularity estimate of $\Phi$ in terms of the the spectral radius $\rho\left(\mathbf{T}_{\mathbb{H}^{0}}\right)$ of $\left.\mathbf{T}\right|_{\mathbb{H}^{0}}$, and therefore we need to find the maximum of the moduli of the eigenvalues of $\left.\mathbf{T}\right|_{\mathbb{H}^{0}}$. Since the product of the left and right eigenvectors of a simple eigenvalue of a matrix is not zero, Theorem 4.6 leads to the following corollary,

Corollary 5.3. If $\lambda^{-\beta}$ with $\beta \in \mathbb{Z}_{+}^{d},|\beta|<k+k_{0}$, is a simple eigenvalue of $\mathcal{T}$ and there exists $\beta^{\prime} \in E_{\beta}$ such that $\mathbf{L}_{\Omega}^{\beta^{\prime}} \neq 0$, then $\lambda^{-\beta}$ is not an eigenvalue of $\mathbf{T}_{\mathbb{H}^{0}}$.

The next proposition provides a way to find the eigenvalues of $\left.\mathbf{T}\right|_{\mathbb{H}^{0}}$. Let $\mathcal{L}_{\Omega}$ be the $r^{2}|[\Omega]|$ by $\left(\begin{array}{c}d+k+k_{0}-1 \\ d\end{array}\right)$ matrix defined by

$$
\mathcal{L}_{\Omega}:=\left(\cdots,{ }^{t}\left(\mathbf{L}_{\Omega}^{\beta}\right), \cdots\right)_{\beta \in \mathbb{Z}_{+}^{d},|\beta| \leq k+k_{0}-1},
$$

and for $j, 1 \leq j \leq r$, let $L_{j}$ and $R_{j}$ be the $r^{2}|[\Omega]|$ by $\left(\begin{array}{c}d+k-1 \\ d\end{array}\right)$ matrices defined by

$$
L_{j}:=\left(\cdots,{ }^{t}\left({ }_{j} \mathbf{l}_{\Omega}^{\alpha}\right), \cdots\right)_{\alpha \in \mathbb{Z}_{+}^{d},|\alpha| \leq k-1}, \quad R_{j}:=\left(\cdots,{ }^{t}\left({ }_{j} \mathbf{r}_{\Omega}^{\alpha}\right), \cdots\right)_{\alpha \in \mathbb{Z}_{+}^{d},|\alpha| \leq k-1} .
$$

Then define the $r^{2}|[\Omega]|$ by $\left(\begin{array}{c}d+k+k_{0}-1 \\ d\end{array}\right)+2 r\left(\begin{array}{c}d+k-1 \\ d\end{array}\right)$ matrix $M_{\Omega}$ by

$$
M_{\Omega}:=\left(\mathcal{L}_{\Omega}, L_{1}, \cdots, L_{r}, R_{1}, \cdots, R_{r}\right) .
$$

Proposition 5.4. Assume that $\lambda_{0}$ is a nonzero eigenvalue of $\mathbf{T}$, . Then $\lambda_{0}$ is an eigenvalue of $\left.\mathbf{T}\right|_{\mathbb{H}^{0}}$ if and only if $\operatorname{rank}\left({ }^{t} M_{\Omega}\left(\mathbf{u}_{1}, \cdots, \mathbf{u}_{l}\right)\right)<l$, where $\mathbf{u}_{1}, \cdots, \mathbf{u}_{l}$ constitute a basis of the $\lambda_{0}$-eigenspace of $\mathcal{T}$.

Proof. Note that $\lambda_{0}$ is a nonzero eigenvalue of $\left.\mathbf{T}\right|_{\mathbb{H}^{0}}$ if and only if $\lambda_{0}$ is a nonzero eigenvalue of $\mathcal{T}$ with a corresponding right eigenvector $\mathbf{u}$ satisfying

$$
{ }^{t} M_{\Omega} \mathbf{u}=0 .
$$

By the fact that for any matrices $M_{1}, M_{2}$ (with the product $M_{1} M_{2}$ meaningful), $\operatorname{rank}\left(M_{1} M_{2}\right) \leq \min \left(\operatorname{rank} M_{1}, \operatorname{rank} M_{2}\right)$, we know that if $\operatorname{rank}\left({ }^{t} M_{\Omega}\left(\mathbf{u}_{1}, \cdots, \mathbf{u}_{l}\right)\right) \geq l$, then $\operatorname{rank}\left({ }^{t} M_{\Omega}\left(\mathbf{u}_{1}, \cdots, \mathbf{u}_{l}\right)\right)=l$, and therefore any linear combinations of $\mathbf{u}_{1}, \cdots, \mathbf{u}_{l}$ does not satisfies (5.3). Thus $\lambda_{0}$ is not an eigenvalue of $\left.\mathbf{T}\right|_{\mathbb{H}^{0}}$.

If $\operatorname{rank}\left({ }^{t} M_{\Omega}\left(\mathbf{u}_{1}, \cdots, \mathbf{u}_{l}\right)\right)=l_{0}<l$, we assume without loss of generality that the rank of ${ }^{t} M_{\Omega}\left(\mathbf{u}_{1}, \cdots, \mathbf{u}_{l_{0}}\right)$ is $l_{0}$. Thus ${ }^{t} M_{\Omega} \mathbf{u}_{j}, j=1, \cdots, l_{0}$, are linearly independent, while ${ }^{t} M_{\Omega} \mathbf{u}_{j}, j=1, \cdots, l_{0}+1$, are linearly dependent. Hence we can find constants $c_{1}, \cdots, c_{l_{0}}$ such that

$$
\mathbf{v}:=c_{1} \mathbf{u}_{1}+\cdots+c_{l_{0}} \mathbf{u}_{l_{0}}+\mathbf{u}_{l_{0}+1}
$$

satisfies (5.3), i.e. $\lambda_{0}$ is an eigenvalue of $\left.\mathbf{T}\right|_{\mathbb{H}^{0}}$ with $H_{0} \in \mathbb{H}$ given by $\operatorname{vec}\left(H_{0}\right)=\mathbf{v}$, with $\mathbf{v}$ being a corresponding eigenvector.

Proposition 5.4 provides an easy way to find eigenvalues of $\left.\mathbf{T}\right|_{\mathbb{H}^{0}}$, and its proof shows how to find the corresponding eigenvector. By Proposition 5.4, we have the following corollary.

Corollary 5.5. The spectral radius $\rho\left(\left.\mathbf{T}\right|_{\mathbb{H}^{0}}\right)$ of $\left.\mathbf{T}\right|_{\mathbb{H}^{0}}$ is the maximum of the moduli of all eigenvalues $\lambda_{0}$ of $\mathcal{T}$ satisfying $\operatorname{rank}\left({ }^{t} M_{\Omega}\left(\mathbf{u}_{1}, \cdots, \mathbf{u}_{l}\right)\right)<l$, where $\mathbf{u}_{1}, \cdots, \mathbf{u}_{l}$ are a basis of the $\lambda_{0}$-eigenspace of $\mathcal{T}$. 
For the next proposition, we need to consider the transition operators on other spaces. Denote $\mathcal{N}:=\max \left(N, k+k_{0}\right)$ and

$$
\Omega_{1}:=\left\{\sum_{j=0}^{\infty} M^{-(j+1)} x_{j}: x_{j} \in[-\mathcal{N}, \mathcal{N}]^{d}, \forall j \in \mathbb{Z}_{+}\right\} .
$$

Let $\mathbb{H}_{\Omega_{1}}$ denote the space of all $r \times r$ matrices with each entry a trigonometric polynomial whose Fourier coefficients are supported in $\left[\Omega_{1}\right]$, and let $\mathbf{T}_{\Omega_{1}}$ denote the operator $\mathbf{T}$ restricted to $\mathbb{H}_{\Omega_{1}}$. Then $\mathbf{T}_{\Omega_{1}}$ is a linear operator on $\mathbb{H}_{\Omega_{1}}$ leaving $\mathbb{H}_{\Omega_{1}}$ and $\mathbb{H}$ invariant, and the representing matrix of $\mathbf{T}_{\Omega_{1}}$ is

$$
\mathcal{T}_{\Omega_{1}}:=\left(\mathcal{A}_{2 i-j}\right)_{i, j \in\left[\Omega_{1}\right]} .
$$

Let $\mathbb{H}_{\Omega_{1}}^{0}$ be the subspace of $\mathbb{H}_{\Omega_{1}}$ defined as follows: $H \in \mathbb{H}_{\Omega_{1}}^{0}$ if and only if $\mathbf{L}_{\Omega_{1}}^{\beta} \operatorname{vec}(H)=0,{ }_{j} \mathbf{l}_{\Omega_{1}}^{\alpha} \operatorname{vec}(H)=0$ and ${ }_{j} \mathbf{r}_{\Omega_{1}}^{\alpha} \operatorname{vec}(H)=0$ for all $\beta, \alpha \in \mathbb{Z}_{+}^{d},|\beta|<$ $k+k_{0},|\alpha|<k, 1 \leq j \leq r$. In this case $\mathbf{L}_{\Omega_{1}}^{\beta},{ }_{j} \mathbf{l}_{\Omega_{1}}^{\alpha}$ and ${ }_{j} \mathbf{r}_{\Omega_{1}}^{\alpha}$ are $1 \times\left(r^{2}\left|\left[\Omega_{1}\right]\right|\right)$ vectors defined by (4.9) and (5.1), respectively, with $\Omega_{1}$ instead of $\Omega$. It can be shown similarly that $\mathbb{H}_{\Omega_{1}}^{0}$ is invariant under $\mathbf{T}_{\Omega_{1}}$, and we let $\left.\mathbf{T}\right|_{\mathbb{H}_{\Omega_{1}}^{0}}$ denote the restriction of $\mathbf{T}_{\Omega_{1}}$ (T) to $\mathbb{H}_{\Omega_{1}}^{0}$. Let $H_{0} \in \mathbb{H}_{\Omega_{1}}$ be defined by

$$
H_{0}(\omega)=\sum_{j=1}^{d}\left(1-\cos \left(\omega_{j}\right)\right)^{k+k_{0}} \mathbf{I}_{r}, \quad \omega={ }^{t}\left(\omega_{1}, \cdots, \omega_{d}\right) \in \mathbb{R}^{d} .
$$

Then $H_{0}(\omega) \in \mathbb{H}_{\Omega_{1}}^{0}$, and thus $\mathbb{H}_{\Omega_{1}}^{0}$ is nontrivial. By Lemma 2.2, the eigenvectors of $\mathbf{T}_{\Omega_{1}}$ corresponding to nonzero eigenvalues are in $\mathbb{H}$. Therefore $\mathbf{T}_{\Omega_{1}}\left(\left.\mathbf{T}\right|_{\mathbb{H}_{\Omega_{1}}^{0}}\right.$ resp.) and the restriction $\left.\mathbf{T}\right|_{\mathbb{H}}$ of $\mathbf{T}$ to $\mathbb{H}\left(\left.\mathbf{T}\right|_{\mathbb{H}^{0}}\right.$ resp.) have the same nonzero eigenvalues. Hence $\rho\left(\left.\mathbf{T}\right|_{\mathbb{H}^{0}}\right)=\rho\left(\left.\mathbf{T}\right|_{\mathbb{H}_{\Omega_{1}}^{0}}\right)$, where $\rho\left(\left.\mathbf{T}\right|_{\mathbb{H}^{0}}\right)$ and $\rho\left(\mathbf{T}_{\mathbb{H}_{\Omega_{1}}^{0}}\right)$ denote the spectral radii of $\left.\mathbf{T}\right|_{\mathbb{H}^{0}}$ and $\left.\mathbf{T}\right|_{\mathbb{H}_{\Omega_{1}}^{0}}$, respectively.

The following proposition is obtained by modifying the proof of Proposition 4.4 in [26] or Proposition 3.3 in [19].

Choose a vector norm on the space $\mathbb{H}_{\Omega_{1}}^{0}$ and define the operator (matrix) norm $\left\|\left.\mathbf{T}\right|_{\mathbb{H}_{\Omega_{1}}^{0}}\right\|$ with respect to this vector norm. Then

$$
\lim _{n \rightarrow \infty}\left\|\left(\mathbf{T}_{\mathbb{H}_{\Omega_{1}}^{0}}\right)^{n}\right\|^{1 / n}=\rho\left(\left.\mathbf{T}\right|_{\mathbb{H}_{\Omega_{1}}^{0}}\right)=\rho\left(\left.\mathbf{T}\right|_{\mathbb{H}^{0}}\right) .
$$

Proposition 5.6. Assume that $\mathbf{P}$ satisfies conditions (3.4) and (4.5), and $\rho\left(\left.\mathbf{T}\right|_{\mathbb{H} 0}\right)$ is the spectral radius of $\left.\mathbf{T}\right|_{\mathbb{H}^{0}}$. Then for any $\epsilon>0$, for the corresponding $(M, \mathbf{P})$ matrix refinable function $\Phi$, there exists a constant $c$ independent of $n$ such that

$$
\int_{\mathbb{D}_{n}}|\widehat{\Phi}(w)|^{2} d w \leq c\left(\rho\left(\left.\mathbf{T}\right|_{\mathbb{H}^{0}}\right)+\epsilon\right)^{n},
$$

where $\mathbb{D}_{n}:={ }^{t} M^{n} \mathbb{T}^{d} \backslash\left({ }^{t} M^{n-1} \mathbb{T}^{d}\right), n \in \mathbb{Z}_{+}$.

Proof. Let $H_{0}(\omega) \in \mathbb{H}_{\Omega_{1}}^{0}$ be defined by (5.4). Since ${ }^{t} M^{-1} \mathbb{T}^{d}$ is a neighborhood of the origin, there exists a positive integer $q$ such that $\frac{1}{q} \mathbb{T}^{d} \subset{ }^{t} M^{-1} \mathbb{T}^{d}$. Note that for $\omega \in \mathbb{D}_{n}, \widehat{\Phi}(\omega)=\Pi_{n}(\omega) \widehat{\Phi}\left({ }^{t} M^{-n} \omega\right)$, and for $\omega \in \mathbb{T}^{d} \backslash\left(\frac{1}{q} \mathbb{T}^{d}\right), H_{0}(\omega) \geq c_{0} \mathbf{I}_{r}$ with $c_{0}=d\left(1-\cos \left(\frac{\pi}{q}\right)\right)^{k+k_{0}}>0$. Thus by the continuity of $\widehat{\Phi}(\omega)$ on $\mathbb{T}^{d}$, we have for any 
positive integer $n$,

$$
\begin{aligned}
& \int_{\mathbb{D}_{n}} \widehat{\Phi}(\omega) \widehat{\Phi}^{*}(\omega) d \omega=\int_{\mathbb{D}_{n}} \Pi_{n}(\omega) \widehat{\Phi}\left({ }^{t} M^{-n} \omega\right) \widehat{\Phi}^{*}\left({ }^{t} M^{-n} \omega\right) \Pi_{n}^{*}(\omega) d \omega \\
& \leq c \int_{\mathbb{D}_{n}} \Pi_{n}(\omega) \Pi_{n}^{*}(\omega) d \omega \leq c \int_{{ }^{t} M^{n} \mathbb{T}^{d} \backslash\left(\frac{1}{q}{ }^{t} M^{n} \mathbb{T}^{d}\right)} \Pi_{n}(\omega) \Pi_{n}^{*}(\omega) d \omega \\
& \leq c \int_{{ }^{t} M^{n} \mathbb{T}^{d} \backslash\left(\frac{1}{q}{ }^{t} M^{n} \mathbb{T}^{d}\right)} \Pi_{n}(\omega) H_{0}\left({ }^{t} M^{-n} \omega\right) \Pi_{n}^{*}(\omega) d \omega \\
& \leq c \int_{\mathbb{R}^{d}} \Pi_{n}(\omega) H_{0}\left({ }^{t} M^{-n} \omega\right) \Pi_{n}^{*}(\omega) d \omega=c \int_{\mathbb{T}^{d}}\left(\mathbf{T}_{\Omega_{1}}^{n} H_{0}\right)(\omega) d \omega,
\end{aligned}
$$

where the last equality follows from Lemma 2.9. Since the Hilbert-Schmidt norm $\|Q\|_{2}=\sqrt{\operatorname{Tr}\left(Q Q^{*}\right)}$ is an equivalent norm for finite matrices, by applying the trace operation, we obtain

$\int_{\mathbb{D}_{n}}|\widehat{\Phi}(\omega)|^{2} d \omega=\int_{\mathbb{D}_{n}} \operatorname{Tr}\left(\widehat{\Phi}(\omega) \widehat{\Phi}^{*}(\omega)\right) d \omega \leq c_{\epsilon}\left(\rho\left(\left.\mathbf{T}\right|_{\mathbb{H}_{\Omega_{1}}^{0}}\right)+\epsilon\right)^{n}=c_{\epsilon}\left(\rho\left(\mathbf{T}_{\mathbb{H}^{0}}\right)+\epsilon\right)^{n}$ with $c_{\epsilon}$ independent of $n$.

Proposition 5.6 together with the usual Littlewood-Paley technique leads to the following Sobolev estimate of the refinable vector $\Phi$.

Theorem 5.7. Assume that $\mathbf{P}$ satisfies (3.4) and (4.5). Then the (M, $\mathbf{P})$ matrix refinable function $\Phi$ is in $W^{s}\left(\mathbb{R}^{d}\right)$ for any $s<s_{0}:=-\log \rho\left(\left.\mathbf{T}\right|_{\mathbb{H}^{0}}\right) /\left(2 \log \lambda_{\max }\right)$, where $\rho\left(\left.\mathbf{T}\right|_{\mathbb{H}^{0}}\right)$ is the spectral radius of $\left.\mathbf{T}\right|_{\mathbb{H}^{0}}$ and $\lambda_{\max }:=\max \left\{\left|\lambda_{1}\right|, \cdots,\left|\lambda_{d}\right|\right\}$.

Proof. For the dilation matrix $M$, there exists some $n_{0} \in \mathbb{Z}_{+}$such that $\mathbb{T}^{d} \subset$ $\left({ }^{t} M\right)^{n_{0}+1} \mathbb{T}^{d}$. For $s<s_{0}$, let $\epsilon>0$ be a constant satisfying

$$
s<-\log \left(\epsilon+\rho\left(\left.\mathbf{T}\right|_{\mathbb{H}^{0}}\right)\right) /\left(2 \log \lambda_{\max }\right) .
$$

Since

$$
\int_{\mathbb{D}_{n}}|\widehat{\Phi}(w)|^{2} d \omega \leq c\left(\epsilon+\rho\left(\left.\mathbf{T}\right|_{\mathbb{H}^{0}}\right)\right)^{n}
$$

for some constant $c$ independent of $n$, and $\widehat{\Phi}$ is continuous on $\mathbb{T}^{d}$, it follows that

$$
\begin{aligned}
& \int_{\mathbb{R}^{d}}\left(1+|\omega|^{2}\right)^{s}|\widehat{\Phi}(\omega)|^{2} d \omega \\
& \leq \int_{\mathbb{T}^{d}}\left(1+|\omega|^{2}\right)^{s}|\widehat{\Phi}(\omega)|^{2} d \omega+\sum_{n=1}^{\infty} \int_{{ }^{t} M^{n_{0}+n} \mathbb{T}^{d} \backslash{ }^{t} M^{n-1} \mathbb{T}^{d}}\left(1+|\omega|^{2}\right)^{s}|\widehat{\Phi}(\omega)|^{2} d \omega \\
& =\int_{\mathbb{T}^{d}}\left(1+|\omega|^{2}\right)^{s}|\widehat{\Phi}(\omega)|^{2} d \omega+\sum_{n=1}^{\infty} \sum_{j=0}^{n_{0}} \int_{\mathbb{D}_{n+j}}\left(1+|\omega|^{2}\right)^{s}|\widehat{\Phi}(\omega)|^{2} d \omega \\
& \leq c+c \sum_{n=1}^{\infty} \sum_{j=0}^{n_{0}}\left(\lambda_{\max }\right)^{2(n+j) s}\left(\epsilon+\rho\left(\left.\mathbf{T}\right|_{\left.\left.\mathbb{H}^{0}\right)\right)^{n}<\infty}\right.\right.
\end{aligned}
$$

Therefore $\Phi \in W^{s}\left(\mathbb{R}^{d}\right)$.

Let $C^{\gamma}\left(\mathbb{R}^{d}\right)$ denote the space defined as the following way: if $\gamma=n+\gamma^{\prime}$ with $n \in \mathbb{Z}_{+}$and $0 \leq \gamma^{\prime}<1$, then $f \in C^{\gamma}\left(\mathbb{R}^{d}\right)$ if and only if $f \in C^{(n)}\left(\mathbb{R}^{d}\right)$ and $f^{(n)}$ is 
uniformly Hölder continuous with exponent $\gamma^{\prime}$, i.e.

$$
\left|D^{\beta} f(x+y)-D^{\beta} f(x)\right| \leq c|y|^{\gamma^{\prime}}, \quad \text { for any } \beta \in \mathbb{Z}_{+}^{d},|\beta|=n,
$$

for some constant $c$ independent of $x, y \in \mathbb{R}^{d}$. With the well-known inclusion

$$
W^{s}\left(\mathbb{R}^{d}\right) \subset C^{\gamma}\left(\mathbb{R}^{d}\right), \quad \text { for } s>\gamma+\frac{d}{2},
$$

Theorem 5.7 leads to the following corollary.

Corollary 5.8. Suppose $\mathbf{P}$ satisfies conditions (3.4) and (4.5). Then the $(M, \mathbf{P})$ matrix refinable function $\Phi \in C^{\gamma}\left(\mathbb{R}^{d}\right)$ for any $\gamma<-\frac{d}{2}-\log \rho\left(\left.\mathbf{T}\right|_{\mathbb{H}^{0}}\right) /\left(2 \log \lambda_{\max }\right)$, where $\rho\left(\left.\mathbf{T}\right|_{\mathbb{H}^{0}}\right)$ is the spectral radius of $\left.\mathbf{T}\right|_{\mathbb{H}^{0}}$ and $\lambda_{\max }:=\max \left\{\left|\lambda_{1}\right|, \cdots,\left|\lambda_{d}\right|\right\}$.

Assume that the refinement mask $\left\{\mathbf{P}_{\alpha}\right\}$ is a finitely supported real $r \times r$ matrix sequence and $\mathbf{P}$ satisfies the vanishing moment conditions of order $k$ (3.4) and (4.5) for some $k_{0}$ with real vectors $\mathbf{l}_{0}^{\beta},|\beta|<k+k_{0}$. Let $\mathbb{H}_{\mathrm{r}}$ denote the space of all $r \times r$ matrices with each entry a trigonometric polynomial whose Fourier coefficients are real and supported in $[\Omega]$. Then $\mathbb{H}_{\mathrm{r}}$ is invariant under $\mathbf{T}$. Define the subspace $\mathbb{H}_{\text {sym }}$ of $\mathbb{H}_{\mathrm{r}}$ by

$$
\begin{aligned}
& \mathbb{H}_{\mathrm{sym}}:=\left\{H \in \mathbb{H}_{\mathrm{r}}: \quad H^{*}=H, \quad \mathbf{L}_{\Omega}^{\beta} \operatorname{vec}(H)=0\right. \text { and } \\
&\left.{ }_{j} \mathbf{l}_{\Omega}^{\alpha} \operatorname{vec}(H)=0, \forall \beta, \alpha \in \mathbb{Z}_{+}^{d},|\beta|<k+k_{0},|\alpha|<k, 1 \leq j \leq r\right\} .
\end{aligned}
$$

Then $\mathbb{H}_{\text {sym }}$ is a linear space over the field $\mathbb{R}$ and is invariant under $\mathbf{T}$. Let $\left.\mathbf{T}\right|_{\mathbb{H}_{\text {sym }}}$ denote the restriction of $\mathbf{T}$ to $\mathbb{H}_{\text {sym }}$. Then, as above, we can obtain the Sobolev regularity estimate of the compactly supported $(M, \mathbf{P})$ refinable vector $\Phi$ in terms of the spectral radius of $\left.\mathbf{T}\right|_{\mathbb{H}_{\mathrm{sym}}}$.

Theorem 5.9. Assume that the refinement mask $\left\{\mathbf{P}_{\alpha}\right\}$ is a finitely supported real $r \times r$ matrix sequence and $\mathbf{P}$ satisfies (3.4) and (4.5) with real vectors $\mathbf{1}_{0}^{\beta},|\beta|<k+k_{0}$. Then the $(M, \mathbf{P})$ matrix refinable function $\Phi$ is in $W^{s}\left(\mathbb{R}^{d}\right)$ for any $s<s_{0}:=$ $-\log \rho\left(\left.\mathbf{T}\right|_{\mathbb{H}_{\text {sym }}}\right) /\left(2 \log \lambda_{\max }\right)$, where $\rho\left(\left.\mathbf{T}\right|_{\mathbb{H}_{\text {sym }}}\right)$ is the spectral radius of $\left.\mathbf{T}\right|_{\mathbb{H}_{\mathrm{sym}}}$ and $\lambda_{\max }:=\max \left\{\left|\lambda_{1}\right|, \cdots,\left|\lambda_{d}\right|\right\}$.

In [19], the Sobolev regularity estimates of the B-splines defined by knots $0,0,1,1$ and $0,1,1,2$, the GHM-orthogonal scaling functions in [8] and two refinable vectors from [2] are analyzed. To finish this paper, we analyze an example from [9] about refinable bivariate splines.

Example 5.10. Let $\phi_{1}$ denote the "pyramid function" with support on the square with vertices $(2,1),(1,2),(0,1)$ and $(1,0)$ which is continuous, satisfies $\phi_{1}(1,1)=1$ and is linear on each of the four triangles formed by the boundary and the two diagonals of its support. Let $\phi_{2}$ be the "pyramid function" with support on $[1,2]^{2}$, i.e.

$$
\phi_{2}\left(x_{1}, x_{2}\right)=\phi_{1}\left(x_{1}+x_{2}-1, x_{1}-x_{2}\right) .
$$

Let $\Phi:={ }^{t}\left(\phi_{1}, \phi_{2}\right)$. Then $\Phi$ satisfies the matrix refinement equations (1.1) with $M=2 \mathbf{I}_{2}$ and the matrix refinement mask given by (refer to [9])

$$
\mathbf{P}(\omega):=\frac{1}{8}\left(\begin{array}{cc}
z_{1}+z_{2}+2 z_{1} z_{2}+z_{1}^{2} z_{2}+z_{1} z_{2}^{2} & \left(1+z_{1}\right)\left(1+z_{2}\right) \\
2\left(z_{1} z_{2}\right)^{2} & z_{1} z_{2}\left(1+z_{1}\right)\left(1+z_{2}\right)
\end{array}\right),
$$


where $z_{1}=e^{-i \omega_{1}}, z_{2}=e^{-i \omega_{2}}$. In this case $\eta_{j}=\gamma_{j}, j=0, \cdots, 3$, and they are the vertices of $[0,1]^{2}$, and $1, \frac{1}{4}$ are eigenvalues of $\mathbf{P}(0), N=2, \Omega=[-2,2]^{2}$. One has

$$
\mathbf{P}(0)=\frac{1}{8}\left(\begin{array}{ll}
6 & 4 \\
2 & 4
\end{array}\right), \quad \mathbf{P}\left(\pi \eta_{j}\right)=\frac{1}{8}\left(\begin{array}{cc}
-2 & 0 \\
2 & 0
\end{array}\right), \quad j=1,2,3 .
$$

Thus $\mathbf{l}_{0}^{(00)}={ }^{t}(1,1)$ is the unique (up to a nonzero constant) vector satisfying (3.4) for $\beta=(00)$, and we have

$$
\begin{aligned}
& D^{(10)} \mathbf{P}(0)=D^{(10)} \mathbf{P}(0)=\frac{-i}{8}\left(\begin{array}{ll}
6 & 2 \\
4 & 6
\end{array}\right), \\
& D^{(10)} \mathbf{P}(\pi, 0)=D^{(01)} \mathbf{P}(0, \pi)=\frac{-i}{8}\left(\begin{array}{cc}
-2 & -2 \\
4 & 2
\end{array}\right), \\
& D^{(10)} \mathbf{P}(0, \pi)=D^{(01)} \mathbf{P}(\pi, 0)=D^{(10)} \mathbf{P}(\pi, \pi)=D^{(01)} \mathbf{P}(\pi, \pi)=\frac{-i}{8}\left(\begin{array}{cc}
-2 & 0 \\
4 & 0
\end{array}\right) .
\end{aligned}
$$

One can obtain that $\mathbf{l}_{0}^{(10)}=\mathbf{l}_{0}^{(01)}={ }^{t}\left(1, \frac{3}{2}\right)$ satisfy (3.4) for $\beta=(10)$ and $\beta=(01)$, respectively, and there are no such vectors $\mathbf{l}_{0}^{\beta}$ that satisfy (3.4) for all $\beta \in \mathbb{Z}_{+}^{2}$ with $|\beta|=2$. Though $\frac{1}{4}$ is an eigenvalue of $\mathbf{P}(0)$, there are vectors $\mathbf{l}_{0}^{(20)}=\mathbf{l}_{0}^{(02)}=$ ${ }^{t}(1,2), \mathbf{l}_{0}^{(11)}={ }^{t}\left(1, \frac{9}{4}\right)$ and $\mathbf{l}_{0}^{(30)}=\mathbf{l}_{0}^{(03)}={ }^{t}\left(1, \frac{9}{4}\right), \mathbf{l}_{0}^{(21)}=\mathbf{l}_{0}^{(12)}={ }^{t}(1,3)$ satisfying $(4.5)$ for $\beta=(20),(02),(30),(03),(21)$ and (12), respectively. To check the stability of $\Phi$, we need to compute the eigenvalues of the $100 \times 100$ matrix

$$
\mathcal{T}_{[-2,2]^{2}}=\left(\mathcal{A}_{2 i-j}\right)_{i, j \in[-2,2]^{2}} .
$$

We find for $\beta \in \mathbb{Z}_{+}^{d},|\beta| \leq 3$, that $\mathbf{L}_{[-2,2]^{2}}^{\beta} \neq 0$. Thus by Theorem $4.2,1, \frac{1}{2}, \frac{1}{4}$ and $\frac{1}{8}$ are eigenvalues of $\mathcal{T}$. In fact the eigenvalues of $\mathcal{T}$ are $1, \frac{1}{2}(2), \frac{1}{4}(5), \frac{1}{8}(12), \frac{1}{16}(24)$ and $0(56)$. Here for an eigenvalue $\lambda_{0}$, the notation $\lambda_{0}(l)$ means that the algebraic multiplicity of $\lambda_{0}$ is $l$. Thus $\mathcal{T}_{[-2,2]^{2}}$ and the transition operator $\mathbf{T}$ restricted to $\mathbb{H}_{[-2,2]^{2}}$, denoted by $\mathbf{T}_{[-2,2]^{2}}$, satisfy Condition E. We find that the 1-eigenvector of $\mathbf{T}_{[-2,2]^{2}}$ is

$$
H(\omega)=\left(\begin{array}{cc}
8+e^{i \omega_{1}}+e^{i \omega_{2}}+e^{-i \omega_{1}}+e^{-i \omega_{2}} & 1+e^{i \omega_{1}}+e^{i \omega_{2}}+e^{i\left(\omega_{1}+\omega_{2}\right)} \\
1+e^{-i \omega_{1}}+e^{-i \omega_{2}}+e^{-i\left(\omega_{1}+\omega_{2}\right)} & 4
\end{array}\right) .
$$

Checking directly, $H(\omega)>0$ for all $\omega \in \mathbb{T}^{2}$; hence $\Phi$ is stable. By Theorem 3.6, $\mathcal{S}(\Phi)$ provides approximation of order 2 .

To estimate the regularity by our method, we need only to find the maximum of the moduli of the eigenvalues of $\left.\mathbf{T}_{[-2,2]^{2}}\right|_{\mathbb{H}^{0}}$, the restriction of $\mathbf{T}_{[-2,2]^{2}}$ to the invariant subspace $\mathbb{H}^{0}$ of $\mathbb{H}_{[-2,2]^{2}}$ defined by (5.2). By Corollary 5.3 and Proposition 5.4 , we find that $1, \frac{1}{2}$ and $\frac{1}{4}$ are not eigenvalues of $\left.\mathbf{T}_{[-2,2]^{2}}\right|_{\mathbb{H}^{0}}$, and $\frac{1}{8}$ is an eigenvalue of $\left.\mathbf{T}_{[-2,2]^{2}}\right|_{\mathbb{H}^{0}}$ with a corresponding eigenvector $H^{0}(\omega)=\sum_{\ell \in[-1,1]^{2}} H_{\ell} e^{-i \ell \omega}$ given by

$$
\begin{aligned}
& H_{-1-1}={ }^{t} H_{11}=\left(\begin{array}{cc}
1 & 4 \\
0 & 0
\end{array}\right), \quad H_{-10}={ }^{t} H_{10}=\left(\begin{array}{cc}
-6 & 6 \\
0 & 0
\end{array}\right), \\
& H_{0-1}={ }^{t} H_{01}=\left(\begin{array}{cc}
0 & 6 \\
0 & -6
\end{array}\right), \quad H_{00}=\left(\begin{array}{cc}
-10 & 4 \\
4 & -8
\end{array}\right),
\end{aligned}
$$

and $H_{-11}={ }^{t} H_{1-1}=\mathbf{0}$. Thus $\rho\left(\left.\mathbf{T}_{[-2,2]^{2}}\right|_{\mathbb{H}^{0}}\right)=\frac{1}{8}$, and it follows from Theorem 5.7 or Theorem 5.9 that $\Phi \in W^{\frac{3}{2}-\epsilon}\left(\mathbb{R}^{2}\right)$ for any $\epsilon>0$. On the other hand, the Fourier 
transform of $\Phi$ is (see [9])

$$
\begin{aligned}
& \widehat{\phi}_{1}\left(\omega_{1}, \omega_{2}\right)=4 e^{-i\left(\omega_{1}+\omega_{2}\right)} \frac{\omega_{1} \sin \omega_{2}-\omega_{2} \sin \omega_{1}}{\omega_{1} \omega_{2}\left(\omega_{1}^{2}-\omega_{2}^{2}\right)}, \\
& \widehat{\phi}_{2}\left(\omega_{1}, \omega_{2}\right)=\frac{1}{2} e^{-\frac{3}{2} i\left(\omega_{1}+\omega_{2}\right)} \widehat{\phi}_{1}\left(\frac{\omega_{1}+\omega_{2}}{2}, \frac{\omega_{1}-\omega_{2}}{2}\right) .
\end{aligned}
$$

Thus $\Phi \in W^{s}\left(\mathbb{R}^{2}\right)$ if and only if $s<\frac{3}{2}$, and our estimate on the Sobolev regularity of $\Phi$ is optimal.

Acknowledgements. The author would like to express his gratitude to Professor S. L. Lee and Dr. Zuowei Shen for generous help and useful suggestions. The author would like to thank Professor Rong-Qing Jia for helpful discussions about approximation order.

\section{REFERENCES}

1. C. Cabrelli, C. Heil and U. Molter, Accuracy of lattice translates of several multidimensional refinable functions, J. Approx. Theory 95 (1998), 5-52. CMP 99:01

2. C. K. Chui and J. Lian, A study on orthonormal multi-wavelets, Appl. Numer. Math., 20 (1996), 273-298. MR 98g:42051

3. A. Cohen, I. Daubechies and G. Plonka, Regularity of refinable function vectors, J. Fourier Anal. and Appl., 3 (1997), 295-324. MR 98e:42031

4. M. Collins, Representations and characters of finite groups, Cambridge Univ. Press, Cambridge, 1990. MR 91f:20001

5. I. Daubechies, Ten Lectures on Wavelets, SIAM, Philadelphia, 1992. MR 93e:42045

6. C. de Boor, R. DeVore and A. Ron, The structure of finitely generated shift-invariant spaces in $L_{2}\left(\mathbb{R}^{d}\right)$, J. Funct. Anal., 119 (1994), 37-78. MR 95g:46050

7. C. de Boor, R. DeVore and A. Ron, Approximation orders of FSI spaces in $L_{2}\left(\mathbb{R}^{d}\right)$, I, II, Constr. Approx. 14 (1998), 411-427, 631-652. CMP 98:14; CMP 99:01

8. J. Geronimo, D. Hardin and P. Massopust, Fractal functions and wavelet expansions based on several scaling functions, J. Approx. Theory 78 (1994), 373-401. MR 95h:42033

9. T. N. Goodman, Pairs of refinable bivariate splines, Advanced Topics in Multivariate Approximation (F. Fontanelle, K. Jetter and L. L. Schumaker, eds.), World Sci. Publ. Co., 1996.

10. T. N. Goodman, S. L. Lee and W. S. Tang, Wavelets in wandering subspaces, Trans. Amer. Math. Soc., 338 (1993), 639-654. MR 93j:42017

11. C. Heil, G. Strang and V. Strela, Approximation by translates of refinable functions, Numer. Math. 73 (1996), 75-94. MR 97c:65033

12. R. Horn and C. Johnson, Topics in Matrix Analysis, Cambridge Univ. Press, Cambridge, 1991. MR 92e: 15003

13. R. Jia, Refinable shift-invariant spaces:from splines to wavelets, Approximation Theory VIII, vol. 2 (C. K. Chui and L. L. Schumaker, eds.), 1995, pp. 179-208. MR 98d:41002

14. R. Jia, Shift-invariant spaces and linear operator equations, Israel J. Math. 103 (1998), 259-288.

15. R. Jia, Characterization of smoothness of multivariate refinable functions in Sobolev spaces, Trans. Amer. Math. Soc. (to appear).

16. R. Jia and C. Micchelli, Using the refinement equation for the construction of prewavelets II: Powers of two, Curves and Surfaces (P. J. Laurent, A. Le Méhauté and L. L. Schumaker, eds.), Academic Press, New York, 1991, pp. 209-246. MR 93e:65024

17. R. Jia, S. Riemenschneider and D. Zhou, Approximation by multiple refinable functions, Canadian J. Math. 49 (1997), 944-962. CMP 98:08

18. R. Jia and Z. Shen, Multiresolution and wavelets, Proc. Edinburgh Math. Soc., 37 (1994), 271-300. MR 95h:42035

19. Q. Jiang, On the regularity of matrix refinable functions, SIAM J. Math. Anal. 29 (1998), 1157-1176. CMP 98:11

20. Q. Jiang and S. L. Lee, Matrix continuous refinement equations, preprint, 1996.

21. Q. Jiang and Z. Shen, On existence and weak stability of matrix refinable functions, Constr. Approx., to appear. 
22. W. Lawton, S. L. Lee and Z. Shen, Stability and orthonormality of multivariate refinable functions, SIAM J. Math. Anal., 28 (1997), 999-1014. MR 98d:41027

23. R. Long, W. Chen and S. Yuan, Wavelets generated by vector multiresolution analysis, Appl. Comput. Harmon. Anal., 4 (1997), 317-350, MR 98m:42052

24. C. Micchelli and T. Sauer, Regularity of multiwavelets, Advances in Comp. Math., 7 (1997), 455-456. CMP 98:01

25. G. Plonka, Approximation order provided by refinable function vectors, Constr. Approx., 13 (1997), 221-244. MR 98c:41023

26. Z. Shen, Refinable function vectors, SIAM J. Math. Anal. 29 (1998), 235-250. CMP 98:11

27. G. Strang and G. Fix, A Fourier analysis of finite-element variational method, Constructive Aspects of Functional Analysis, (G. Geymonat ed.), C.I.M.E, 1973, pp. 793-840.

28. L. Villemoes, Energy moments in time and frequency for two-scale difference equation solutions and wavelets, SIAM J. Math. Anal., 23 (1992), 1519-1543. MR 94e:39002

Department of Mathematics, National University of Singapore, 10 Kent Ridge Crescent, Singapore 119260 and Department of Mathematics, Peking University, Beijing 100871, CHINA

E-mail address: qjiang@haar.math.nus.edu.sg 